Supporting Information

\title{
Trophic magnification of organic chemicals: a global synthesis
}

Short Title: Global patterns of organic chemical biomagnification

D.M. Walters, ${ }^{*}{ }^{\dagger}$ T.D. Jardine, ${ }^{\dagger}$ B.S. Cade ${ }^{\dagger}$ K.A. Kidd ${ }^{\S}$, D.C.G. Muir, ${ }^{\Perp}$ and P. Leipzig-Scott ${ }^{\dagger}$

${ }^{\dagger}$ U.S. Geological Survey, Fort Collins Science Center, 2150 Centre Avenue, Building C, Fort Collins, Colorado 80526, United States

Hniversity of Saskatchewan, Toxicology Centre, 44 Campus Drive, Saskatoon, Saskatchewan S7N 5B3, Canada

${ }^{\S}$ Canadian Rivers Institute and Biology Department, University of New Brunswick, 100 Tucker Park Road, Saint John, New Brunswick E2L 4L5, Canada

$\|_{\text {Environment and Climate Change Canada, Aquatic Contaminants Research Division, }}$ Burlington ON L7S 1A1, Canada

*Corresponding author: phone: 970-226-9484, fax: 970-226-9230; email: waltersd@usgs.gov 


\section{Supporting Information}

\section{SI Methods}

\section{Calculating Trophic Magnification Factors (TMFs)}

Early assessments of contaminant biomagnification in aquatic food webs were based upon relationships between chemical concentrations and $\delta^{15} \mathrm{~N}$, where $\delta^{15} \mathrm{~N}$ was used as a measure of relative trophic level (TL) within a food web, using the equation:

$$
\text { ln or } \log _{10}[\mathrm{POP}]=b+\left(m \times \delta^{15} \mathrm{~N}\right)
$$

Here, a significant and positive slope of the regression (the trophic magnification slope, TMS; 1 ) indicates biomagnification. Later, $\delta^{15} \mathrm{~N}$ was replaced with TL based upon the $\delta^{15} \mathrm{~N}$ value of primary producers or consumers in the food web using the equations:

$$
\begin{aligned}
\mathrm{TL}_{\text {consumer }} & =\left[\left(\delta^{15} \mathrm{~N}_{\text {consumer }}-\delta^{15} \mathrm{~N}_{\text {primary producer }}\right) / 3.4\right]+1 \\
\mathrm{TL}_{\text {consumer }} & =\left[\left(\delta^{15} \mathrm{~N}_{\text {consumer }}-\delta^{15} \mathrm{~N}_{\text {primary consumer }}\right) / 3.4\right]+2
\end{aligned}
$$

These assume that primary producers (eq. 2) or consumers (eq. 3) occupy discrete TLs of 1 or 2, respectively $(2,3)$. Substituting TL into eq. 1 then yields the following equation:

$$
\ln \text { or } \log _{10}[\mathrm{POP}]=b+(m \times \mathrm{TL})
$$

The TMF (also called a food web magnification factor, FWMF; 3, 4) is calculated as the antilog of the regression slope with base 10 or $e$ (depending on the logarithmic transformation of the contaminant concentration) using the equation:

$$
\mathrm{TMF}=e^{\mathrm{m}} \text { or } 10^{m}
$$

An advantage of TMF over a $\delta^{15} \mathrm{~N}$-derived slope is that it corrects for baseline variation in $\delta^{15} \mathrm{~N}$ among systems (3). This allows for cross-system comparisons to determine how biomagnification varies according to the properties of ecosystems or the chemical of interest (2). Organic contaminant concentrations are typically lipid normalized before calculating TMF because apparent biomagnification can occur due simply to partitioning of hydrophobic compounds between low lipid prey and high lipid predators. A TMF $>1$ indicates biomagnification, on average, through the food web, and a TMF $<1$ indicates no biomagnification or trophic dilution.

\section{Data Acquisition and Database Construction}

We searched the online databases Web of Knowledge and Google Scholar to find articles on food webs and organic contaminants using the terms biomagnification, bioaccumulation, nitrogen isotope, $\delta^{15} \mathrm{~N}$, organic contaminants, organic pollutants, trophic magnification factor, $T M F$, and bioaccumulation. This search included reports published prior to June 2013. In addition, we also examined papers citing early TMF methods papers, as well as gathering 
unpublished data known personally to the authors. All data necessary for equations 1-5 were extracted directly from publications. Many recent publications reported TMFs calculated using eq 5 and $\log _{10}$ lipid normalized concentration data, whereas many older papers reported TMFs using $\ln$ transformed lipid normalized data or a TMS (eq 1). In those cases, we used simple transformations to recalculate TMF on a $\log _{10}$ basis or calculated TMF ourselves using eq $2-5$. In these cases, we assumed a trophic enrichment factor of 3.4\% (eq 2,3) for $\delta^{15} \mathrm{~N}$ unless a study provided a food web-specific value. We excluded those studies that only calculated TMF or TMS using wet or dry weight contaminant concentrations if they did not also include lipid data (5), but retained studies even if the range of TLs in the food web was limited, provided multiple taxa were included. We also included studies where $\log$ [contaminant] vs. TL regressions were non-significant, while recognizing that type 2 errors (falsely concluding that $\mathrm{TMF}<1$ ) can occur in cases where sample sizes are low and/or variability is high $(2,6,7)$. We excluded studies on perfluorinated compounds (e.g., 8 ) because these compounds associate with protein rather than lipid and thus cannot be lipid-corrected, creating challenges in modelling TMFs with $\log K_{\text {Ow. }}$.

$K_{O W}$ s reported in journal articles were used in our database, with some exceptions. $K_{O W}$ s for polychlorinated biphenyls (PCBs) were taken from Hawker and Connell (9) and Zhou et al. (10), and $K_{O W}$ S for polybrominated diphenyl ethers were from Kelly et al. (11). $K_{O W}$ s for siloxanes were calculated using KOWWIN applications within the U.S. EPA's EPISuite 4.1 (Estimations Program Interface; http://www.epa.gov/oppt/exposure/pubs/ episuite.htm). $K_{O W} \mathrm{~S}$ for toxaphene related compounds were taken from Fisk et al. (12) or calculated using the SMILES notations in EPISuite. $K_{O W}$ f for some compounds were not reported in the individual food web studies or elsewhere in the literature. In these cases, we retrieved $K_{\text {Ow }}$ from online databases ChemSpider (http://www.chemspider.com/) and PubChem (https://pubchem.ncbi.nlm.nih.gov/). When these databases included both experimentally measured and predicted $K_{O W} \mathrm{~s}$ for some compounds, we used the measured $K_{\text {OW }}$. Many articles reported TMFs for co-eluting compounds. We included these in our database if the difference in their $K_{O W} \mathrm{~s}$ was $<0.5 \log$ units, and we calculated a mean $K_{O W}$ among these compounds. These latter values were retrieved from the articles themselves or articles cited therein, from other publications reporting $\log K_{\mathrm{OW}}$ for whole classes of compounds, and from online chemical databases. Compound-specific $k_{\mathrm{M}}$, values were standardized for a 10 gram fish, obtaining median estimates from a summary database (13) when possible and the BCFBAF function in EPIsuite 4.1 to estimate the remainder, drawing structures as necessary. These methods resulted in $n=1,533 \mathrm{TMF}$ values with both $\log$ $K_{\mathrm{OW}}$ and $k_{\mathrm{M}}$ values.

Testing effects of latitude and food web type on TMF. Previous studies have shown significantly higher TMFs at higher latitudes for certain compounds (14). Given higher food consumption rates in birds and mammals relative to fish and invertebrates, any latitude effects could also be more prominent in food webs containing these taxa. We therefore simultaneously tested the effects of latitude and food web type ("cold" vs. "whole") on TMFs for a select number of compounds from our database.

From the larger database, we pulled out compounds that had sufficient sample sizes for both "cold" and "whole" food webs. We used a minimum sample size of $\mathrm{n}=7$ within a food web type as criteria for inclusion. In order to reduce bias from a single study, we combined and averaged three TMFs calculated for a single food web in different seasons (15). This left us with 
five compounds: DDE and four PCB congeners (PCB-118, PCB-138, PCB-153, PCB-180). We ran analyses of covariance (ANCOVA) with food web type as the fixed factor and latitude as the covariate. Because "whole" food webs were fewer and limited the number of comparisons, we additionally ran ordinary least squares regressions of log TMF vs. latitude for "cold" food webs only. An additional 35 compounds had sufficient sample sizes $(n \geq 7)$ to allow inclusion in this analysis. For all comparisons, we excluded studies from the southern hemisphere because of their scarcity in the dataset.

In addition, we constructed simple scatter plots of chemicals that were measured in both cold and warm food webs. We first ran an analysis only on chemicals that included at least $n=5$ observations in both food web categories, and then ran a second, less conservative analysis including all chemicals with $\mathrm{n}=1$ for both categories. The results of these analyses yielded nearly identical trends of higher TMFs in whole food webs.

\section{SI Results}

Effects of latitude and food web type on TMF. We found little effect of latitude on TMF. ANCOVA models showed that TMFs were consistently higher in whole food webs than cold food webs, regardless of latitude (Figure S9). Of the 56 regressions run on cold webs, only seven were significant (without applying a Bonferroni correction) (Table S2). Of those seven, three had negative coefficients which would indicate lower TMFs nearer the poles, the opposite of what we would predict. Across a wide range of compounds, whole food webs had consistently higher TMFs than cold food webs, and this was particularly true for many polychlorinated biphenyls and organochlorine pesticides (Figure S10). 
Table S1. $\log _{10} k_{\mathrm{M}}, \log _{10} K_{O W}$, and mean TMF ( $\pm 1 \mathrm{SD}, \mathrm{n}$ ) for organic chemicals included in the meta-analysis. Numbers in the citation column appear in the References section below.

\begin{tabular}{lclll}
\hline Chemical class and compound & $\log _{10} k_{\mathrm{M}}$ & $\log _{10} K_{O W}$ & TMF & Citation \\
\hline Alkylphenols & & & & \\
$\quad$ Nonylphenol & -0.28 & 5.76 & $0.81(1)$ & $(16)$ \\
$\quad$ Octylphenol & -0.21 & 5.5 & $0.24(1)$ & $(17)$ \\
Brominated Flame Retardants & & & & \\
alpha (+/-) HBCD & -2.26 & 5.2 & $1.53(0.95,6)$ & $(18-21)$ \\
BTBPE & -3.73 & 7.88 & $0.69(0.4,2)$ & $(21,22)$ \\
Hexabromobenzene & -0.59 & 6.07 & $1.76(0.42,2)$ & $(21,23)$ \\
PBT & -0.03 & 6.99 & $0.61(1)$ & $(21)$
\end{tabular}

\section{Chlorinated Flame Retardants}

Anti-dechlorane Plus

$-4.9 \quad 9.03 \quad 3.67(4.35,6) \quad(22,24)$

\section{Chlorinated Paraffins}

$\begin{array}{lllll}\mathrm{C} 10 \mathrm{Cl} 5 & -1.12 & 6.15 & 1.08(0.45,2) & (25) \\ \mathrm{C} 10 \mathrm{Cl} 6 & -1.23 & 6.34 & 0.76(0.2,2) & (25) \\ \mathrm{C} 10 \mathrm{Cl} 7 & -1.34 & 6.52 & 0.9(0.28,2) & (25) \\ \mathrm{C} 10 \mathrm{Cl} 8 & -1.46 & 6.7 & 1.55(0.07,2) & (25) \\ \mathrm{C} 10 \mathrm{Cl} 9 & -1.58 & 6.95 & 1.35(0.07,2) & (25) \\ \mathrm{C} 11 \mathrm{Cl} 10 & -1.84 & 7.62 & 2.4(1) & (25) \\ \mathrm{C} 11 \mathrm{Cl} 5 & -1.26 & 6.65 & 0.54(0.1,2) \\ \mathrm{C} 11 \mathrm{Cl} 6 & -1.37 & 6.83 & 0.57(0.09,2) & (25) \\ \mathrm{C} 11 \mathrm{Cl} 7 & -1.48 & 7.01 & 0.77(0.09,2) \\ \mathrm{C} 11 \mathrm{Cl} 8 & -1.59 & 7.19 & 1.5(0,2) & (25) \\ \mathrm{C} 11 \mathrm{Cl} 9 & -1.71 & 7.37 & 1.5(0.42,2) & (25) \\ \mathrm{C} 12 \mathrm{Cl} 10 & -1.98 & 8.11 & 1.7(1) & (25) \\ \mathrm{C} 12 \mathrm{Cl} 6 & -1.51 & 7.32 & 0.83(0.09,2) & (25)\end{array}$




\begin{tabular}{|c|c|c|c|c|}
\hline $\mathrm{C} 12 \mathrm{Cl} 7$ & -1.62 & 7.5 & $0.98(0.01,2)$ & $(25)$ \\
\hline $\mathrm{C} 12 \mathrm{Cl} 8$ & -1.73 & 7.68 & $1.1(0.14,2)$ & $(25)$ \\
\hline $\mathrm{C} 12 \mathrm{Cl} 9$ & -1.85 & 7.86 & $1.4(0.14,2)$ & $(25)$ \\
\hline $\mathrm{C} 13 \mathrm{Cl} 7$ & -1.76 & 8.05 & $0.58(0.24,2)$ & $(25)$ \\
\hline $\mathrm{C} 13 \mathrm{Cl} 8$ & -1.87 & 8.17 & $0.91(0.41,2)$ & (25) \\
\hline $\mathrm{C} 13 \mathrm{Cl} 9$ & -1.99 & 8.35 & $1.03(0.24,2)$ & $(25)$ \\
\hline $\mathrm{C} 14 \mathrm{Cl} 10$ & -2.24 & 9.02 & 0.21 & $(25)$ \\
\hline $\mathrm{C} 14 \mathrm{Cl} 5$ & -1.74 & 8.12 & $0.31(1)$ & $(25)$ \\
\hline $\mathrm{C} 14 \mathrm{Cl} 6$ & -1.86 & 8.3 & $0.28(1)$ & $(25)$ \\
\hline $\mathrm{C} 14 \mathrm{Cl} 7$ & -1.93 & 8.48 & $0.36(1)$ & $(25)$ \\
\hline $\mathrm{C} 14 \mathrm{Cl} 8$ & -2.01 & 8.66 & $0.29(1)$ & $(25)$ \\
\hline $\mathrm{C} 14 \mathrm{Cl} 9$ & -2.12 & 8.84 & $0.26(1)$ & (25) \\
\hline $\mathrm{C} 15 \mathrm{Cl} 10$ & -2.38 & 9.51 & $0.09(1)$ & $(25)$ \\
\hline $\mathrm{C} 15 \mathrm{Cl} 5$ & -1.88 & 8.61 & $0.3(1)$ & $(25)$ \\
\hline $\mathrm{C} 15 \mathrm{Cl} 6$ & -1.99 & 8.79 & $0.25(1)$ & $(25)$ \\
\hline $\mathrm{C} 15 \mathrm{Cl} 7$ & -2.07 & 8.97 & $0.23(1)$ & $(25)$ \\
\hline $\mathrm{C} 15 \mathrm{Cl} 8$ & -2.19 & 9.15 & 0.11 & $(25)$ \\
\hline $\mathrm{C} 15 \mathrm{Cl} 9$ & -2.26 & 9.33 & $0.09(1)$ & $(25)$ \\
\hline $\mathrm{C} 16 \mathrm{Cl} 7$ & -2.25 & 9.46 & $0.22(1)$ & $(25)$ \\
\hline $\mathrm{C} 16 \mathrm{Cl} 8$ & -2.36 & 9.64 & $0.06(1)$ & $(25)$ \\
\hline
\end{tabular}

\section{Cyclic Methyl Siloxanes}

$\begin{array}{lllll}\text { CVMS-D4 } & -2.04 & 6.79 & 0.64(0.21,5) & (26-28) \\ \text { CVMS-D5 } & -2.97 & 8.03 & 1.47(1.39,6) & (26-29) \\ \text { CVMS-D6 } & -2.5 & 7.35 & 1.08(1.07,5) & (26-28)\end{array}$

Natural Brominated Compounds

$\begin{array}{lllll}2,4,6-\text { Tribromoanisole } & -1.37 & 4.48 & 0.9(1) & (30) \\ \text { Br7-MBP-79 } & -2.4 & 8.3 & 82.0(1) & (31) \\ \text { DBP-Br3Cl3 } & -2.37 & 6.9 & 5.2(1)\end{array}$




$\begin{array}{lllll}\text { DBP-Br4Cl2 } & -2.35 & 7.0 & 14.6(1) & (32) \\ \text { DBP-Br6 } & -2.31 & 7.3 & 7.0(1) & (32) \\ \text { DMP-Br5Cl } & -2.33 & 7.2 & 6.9(1) & (32) \\ \text { MBP-Br5Cl-d } & -2.22 & 7.2 & 15.0(1) & (31) \\ \text { MBP-Br5Cl-e } & -2.22 & 7.2 & 18.0(1) & (31) \\ \text { MBP-Br6-b } & -2.2 & 7.4 & 22.0(1) & (31) \\ \text { MBP-Br6Cl-b } & -2.42 & 8.1 & 25.0(1) & (31) \\ \text { TetraBHD } & -1.56 & 8.08 & 3.4(1) & (30) \\ \text { TriBHD } & -1.35 & 7.19 & 4.2(1) & (30)\end{array}$

\section{Organochlorine Pesticides}

Aldrin

$-2.03$

6.5

$1.91(1)$

(33)

$\underline{\text { Toxaphene Congeners }}$

$\begin{array}{lllll}\text { B6-923 } & -1.92 & 5.69 & 0.83(1) & (34) \\ \text { B7-1001 } & -2.17 & 5.4 & 1.13(1) & (34) \\ \text { B7-515 } & -1.74 & 5.23 & 1.1(1) & (34) \\ \text { B8-1413 } & -2.12 & 5.52 & 0.88(1) & (34) \\ \text { B8-1414/1945 } & -2.13 & 5.38 & 1.36(1) & (34) \\ \text { B8-2229 } & -1.83 & 5.75 & 1.31(1) & (34) \\ \text { B8-531 } & -2.09 & 5.74 & 1.32(1) & (34) \\ \text { B8-789 } & -1.78 & 5.75 & 1.43(1) & (34) \\ \text { B8-806/809 } & -1.97 & 5.78 & 1.48(1) & (34) \\ \text { B9-1025 } & -2.28 & 5.96 & 1.66(1) & (34) \\ \text { B9-1049 } & -2.02 & 5.77 & 1.5(1) & (34) \\ \text { B9-1679 } & -2.3 & 5.84 & 1.29(1) & (34) \\ \text { B9-2206 } & -2.39 & 5.93 & 1.47(1) & (34) \\ \text { B9-715 } & -2.78 & 6.26 & 0.63(1) & (34) \\ \text { Chlordane } & -2.9 & 6.16 & 2.3(0.87,5) & (33,35) \\ \text { Cis-chlordane } & -2.03 & 6.16 & 2.08(1.02,27) & (14,15,33,35-40)\end{array}$




\begin{tabular}{|c|c|c|c|c|}
\hline Cis-nonachlor & -2.5 & 6.08 & $4.12(1.95,7)$ & $(15,35,36,38,39)$ \\
\hline DDMU & -2.06 & 5.5 & $4.6(1.59,2)$ & $(16,35)$ \\
\hline DDT & -2.35 & 6.91 & $1.64(0.39,8)$ & $(23,33,36,38,40-43)$ \\
\hline Dieldrin & -1.88 & 5.4 & $1.58(0.56,20)$ & $(14,33,35,39)$ \\
\hline Endosulfan sulfate & -0.8 & 3.66 & $1.76(1)$ & $(33)$ \\
\hline Endrin aldehyde & -1.74 & 5.73 & $0.83(1)$ & $(33)$ \\
\hline Heptachlor & -1.61 & 5.47 & $0.33(1)$ & (39) \\
\hline Heptachlor-epox & -1.69 & 4.98 & $4.23(4.93,2)$ & $(39,44)$ \\
\hline Lindane & -1.63 & 3.72 & $1.23(0.49,27)$ & $(14,35-37,39-42,45-47)$ \\
\hline Nonachlor-III & -2.5 & 6.06 & $3.06(1)$ & $(35)$ \\
\hline$o, p^{\prime}-D D D$ & -1.81 & 5.87 & $2.2(1)$ & (43) \\
\hline o,p'-DDE & -2.4 & 6.0 & $2.18(0.11,2)$ & $(35,43)$ \\
\hline$o, p^{\prime}-\mathrm{DDT}$ & -1.87 & 6.79 & $2.01(0.01,2)$ & $(40,43)$ \\
\hline Oxychlordane & -1.8 & 5.48 & $10.31(9.63,9)$ & $(15,35-39,48)$ \\
\hline $\mathrm{p}, \mathrm{p}^{\prime}-\mathrm{DDD}$ & -2.6 & 6.02 & $1.81(0.63,7)$ & $(23,33,38,40,43,46,47)$ \\
\hline $\mathrm{p}, \mathrm{p}^{\prime}-\mathrm{DDE}$ & -1.87 & 6.51 & $4.32(3.59,40)$ & $\begin{array}{l}(14-16,23,26,29,33-41 \\
43,46-50)\end{array}$ \\
\hline Tris(4-chlorophenyl)-methane & -2.74 & 7.3 & $2.28(1)$ & $(38)$ \\
\hline Toxaphene & -2.5 & 8.08 & $4.24(4.32,5)$ & $(34,39,51)$ \\
\hline Trans-chlordane & -2.01 & 6.16 & $2.20(3.07,6)$ & $(33,35-37,39,40)$ \\
\hline Nonachlor & -2.5 & 6.35 & $3.55(1.37,28)$ & $\begin{array}{l}(14,15,35,36,38-40,46 \\
48,50)\end{array}$ \\
\hline$\alpha$-endosulfan & -0.89 & 3.83 & $1.52(0.63,5)$ & $(14,33)$ \\
\hline$\alpha-\mathrm{HCH}$ & -1.51 & 3.72 & $1.57(0.89,30)$ & $\begin{array}{l}(14,15,33,35-37,39-41 \\
45,46,50)\end{array}$ \\
\hline$\beta$-endosulfan & -0.89 & 3.83 & $1.04(1)$ & $(33)$ \\
\hline$\beta-\mathrm{HCH}$ & -1.6 & 3.72 & $2.15(1.13,8)$ & $(33,35-38,40,41,45)$ \\
\hline \multicolumn{5}{|c|}{ harmaceuticals and Personal Care Products } \\
\hline AHTN & -1.21 & 5.8 & $0.74(1)$ & $(52)$ \\
\hline
\end{tabular}



HHCB
$-0.71$
5.43
1.12 (1)

(52)

Phthalates

$\begin{array}{lllll}\text { Dibutyl phthalate } & 0.25 & 4.58 & 0.7(1) & (53) \\ \text { Diethyl phthalate } & 1.55 & 2.77 & 1.05(1) & (53) \\ \text { Diisobutyl phthalate } & 1.07 & 4.58 & 0.81(1) & (53) \\ \text { Dimethyl phthalate } & 1.78 & 1.8 & 0.77(1) & (53) \\ \text { Dinonyl phthalate } & -0.54 & 9.11 & 0.28(1) & (53) \\ \text { Dioctyl phthalate } & -0.12 & 8.2 & 0.29(1) & (53) \\ \text { DPE-BBP } & -0.31 & 5.03 & 0.77(1) \\ \text { DPE-C10 } & 0.31 & 10.0 & 0.44(1) \\ \text { DPE-C6 } & 0.4 & 6.39 & 0.98(1) \\ \text { DPE-C7 } & -0.29 & 7.3 & 0.91(1) & (53) \\ \text { DPE-C8 } & -0.16 & 8.2 & 0.5(1) & (53) \\ \text { DPE-C9 } & 0.3 & 9.11 & 0.46(1) & (53) \\ \text { DPE-DEHP } & -0.51 & 8.2 & 0.34(1) & (53)\end{array}$

Polybrominated Diphenyl Ethers

$\begin{array}{lllll}\text { 2'-MeO-BDE-68 } & -2.36 & 7.3 & 2.8(0.71,2) & (11,30) \\ \text { 2'-MeO-BDE-28 } & -1.37 & 6.4 & 0.75(1) & (11) \\ \text { 2'-OH-PBDE-68 } & -0.79 & 6.3 & 0.15(1) & (54) \\ \text { 3-OHPBDE-47 } & -0.79 & 6.77 & 0.27(1) & (54) \\ \text { 6'-MeO-BDE-49 } & -2.36 & 7.3 & 0.8(1) & (11) \\ \text { 6'-MeO-BDE-66 } & -1.57 & 7.3 & 0.98(1) & (11) \\ \text { 6-MeO-PBDE-47 } & -1.57 & 7.3 & 2.5(0.14,2) & (11,30) \\ \text { 6-OH-PBDE-47 } & -0.79 & 6.3 & 0.21(1) & (54) \\ \text { PBDE-15 } & -1.57 & 5.83 & 1.09(0.01,2) & (55,56) \\ \text { PBDE-17 } & -2.12 & 5.74 & 0.98(0.59,2) & (56,57) \\ \text { PBDE-28 } & -2.12 & 5.88 & 1.76(0.96,15) & (11,15,18,23,30,55-62) \\ \text { PBDE-47 } & -2.1 & 6.77 & 3.19(1.88,22) & (11,15,18,20,23,26,29, \\ \end{array}$




\begin{tabular}{|c|c|c|c|c|}
\hline PBDE-49 & -2.1 & 6.8 & $2.99(4.41,4)$ & $(11,18,30,56)$ \\
\hline PBDE-66 & -2.1 & 6.77 & $1.75(0.7,9)$ & $(23,30,56-62)$ \\
\hline PBDE-71 & -2.1 & 6.77 & $1.44(1,2)$ & $(55,59)$ \\
\hline PBDE-77 & -2.1 & 6.73 & $0.8(1)$ & (62) \\
\hline PBDE-85 & -1.58 & 6.71 & $1.7(0.36,4)$ & $(30,57,58,60)$ \\
\hline PBDE-99 & -2.52 & 7.32 & $2.5(2.02,20)$ & $\begin{array}{l}(15,18,23,26,29,30,42 \\
55-62,64)\end{array}$ \\
\hline PBDE-100 & -0.79 & 7.24 & $2.98(1.83,17)$ & $\begin{array}{l}(11,15,18,23,30,47,55- \\
62,64)\end{array}$ \\
\hline PBDE-101 & -2.18 & 7.7 & $0.92(1)$ & $(56)$ \\
\hline PBDE-118 & -2.52 & 7.7 & $3.85(1)$ & $(62)$ \\
\hline PBDE-119 & -2.52 & 6.71 & $3.2(0.84,2)$ & $(59,62)$ \\
\hline PBDE-138 & -2.72 & 7.91 & $1.3(0.62,4)$ & $(18,57,60,62)$ \\
\hline PBDE-153 & -2.15 & 7.9 & $2.16(1.47,12)$ & $\begin{array}{l}(11,18,23,30,55-58,60- \\
62,64)\end{array}$ \\
\hline PBDE-154 & -2.72 & 7.82 & $2.45(1.16,14)$ & $\begin{array}{l}(11,15,23,30,55-58,60- \\
62,64)\end{array}$ \\
\hline PBDE-154/BB153 & -2.72 & 7.82 & $1.53(1)$ & (18) \\
\hline PBDE-183 & -2.92 & 7.49 & $1.19(0.39,5)$ & $(23,57,58,60,62)$ \\
\hline PBDE-190 & -1.66 & 7.49 & $1.88(1)$ & (57) \\
\hline PBDE-196 & -3.12 & 7.9 & $1.99(1)$ & $(60)$ \\
\hline PBDE-197 & -3.12 & 7.9 & $1.1(1)$ & $(60)$ \\
\hline PBDE-203 & -3.12 & 7.9 & $2.94(1)$ & $(60)$ \\
\hline PBDE-205 & -3.12 & 7.9 & $4.47(1)$ & $(60)$ \\
\hline PBDE-206 & -3.32 & 8.3 & $2.85(1)$ & $(60)$ \\
\hline PBDE-207 & -3.32 & 8.3 & $2.13(1)$ & $(60)$ \\
\hline PBDE-208 & -3.32 & 8.3 & $2.5(1)$ & $(60)$ \\
\hline PBDE-209 & -1.23 & 8.7 & $0.32(0.27,5)$ & $(18,20,57,60,64)$ \\
\hline \multicolumn{5}{|c|}{ olychlorinated Benzenes } \\
\hline $\mathrm{HCB}$ & -2.66 & 5.73 & $3.41(3.04,33)$ & $(14,15,18,36-40,42,43$ \\
\hline
\end{tabular}


$46,49,65,66)$

$\begin{array}{lllll}\text { PentaClBz } & -2.4 & 5.18 & 2.93(1) & \text { (36) } \\ \text { TriClBz } & -1.54 & 4.05 & 1.91(1) & \text { (36) }\end{array}$

Polychlorinated Dibenzodioxins and Dibenzofurans

\begin{tabular}{|c|c|c|c|c|}
\hline 1,2,3,4,6,7,8-HpCDD & -1.37 & 6.92 & $0.37(0.27,7)$ & $(67-72)$ \\
\hline 1,2,3,4,6,7,8-HpCDF & -1.41 & 7.92 & $0.38(0.22,9)$ & $(67-72)$ \\
\hline 1,2,3,4,6,7,9-HpCDD & -1.87 & 6.92 & $0.02(1)$ & $(70)$ \\
\hline 1,2,3,4,6,7,9-HpCDF & -1.5 & 8.23 & $0.23(1)$ & $(70)$ \\
\hline 1,2,3,4,6,8,9-HpCDF & -1.5 & 8.23 & $0.18(1)$ & $(70)$ \\
\hline 1,2,3,4,6,8-HxCDD & -1.65 & 6.28 & $0.02(1)$ & $(70)$ \\
\hline $1,2,3,4,6,8-\mathrm{HxCDF}$ & -1.28 & 7.58 & $0.11(1)$ & $(70)$ \\
\hline 1,2,3,4,7,8,9-HpCDF & -1.41 & 7.92 & $0.48(0.43,7)$ & $(67-70,72)$ \\
\hline $\begin{array}{l}1,2,3,4,7,8 / 1,2,3,4,7,9- \\
\text { HxCDF }\end{array}$ & -1.28 & 7.58 & $0.72(0.15,3)$ & $(67,72)$ \\
\hline 1,2,3,4,7,8-HxCDD & -1.43 & 6.28 & $0.47(0.22,8)$ & $(67-72)$ \\
\hline $1,2,3,4,7,8-\mathrm{HxCDF}$ & -1.28 & 7.58 & $0.45(0.23,4)$ & $(68-70)$ \\
\hline 1,2,3,6,7,8-HxCDD & -1.37 & 8.21 & $0.71(0.31,7)$ & $(67-70,72)$ \\
\hline $1,2,3,6,7,8-\mathrm{HxCDF}$ & -1.28 & 7.92 & $0.64(0.34,7)$ & $(67-70,72)$ \\
\hline 1,2,3,6,8-PeCDD & -1.07 & 6.53 & $0.05(1)$ & $(70)$ \\
\hline 1,2,3,7,8,9-HxCDD & -1.46 & 8.21 & $0.51(0.25,7)$ & $(67-70,72)$ \\
\hline 1,2,3,7,8,9-HxCDF & -1.28 & 7.92 & $0.76(0.36,4)$ & $(67,68,72)$ \\
\hline 1,2,3,7,8-PeCDD & -1.72 & 6.64 & $0.94(0.45,8)$ & $(67-72)$ \\
\hline 1,2,3,7,8-PeCDF & -1.01 & 7.27 & $1.63(1.21,4)$ & $(68,69,71)$ \\
\hline 1,2,3,7,9-PeCDD & -1.07 & 6.53 & $0.04(1)$ & $(70)$ \\
\hline 1,2,4,6,8,9-HxCDF & -1.28 & 7.58 & $1.03(1)$ & $(70)$ \\
\hline 1,2,4,6,9-PeCDD & -1.43 & 5.79 & $0.17(1)$ & $(70)$ \\
\hline 1,2,7,8-TeCDF & -0.82 & 6.31 & $0.22(0.24,2)$ & $(70,71)$ \\
\hline 1,3,6,7,8-PeCDF & -0.95 & 6.59 & $0.09(1)$ & $(70)$ \\
\hline 1,3,6,8-TeCDD & -0.95 & 5.29 & $0.26(0.23,2)$ & $(70,71)$ \\
\hline
\end{tabular}




\begin{tabular}{|c|c|c|c|c|}
\hline $1,3,6,8-\mathrm{TeCDF}$ & -0.95 & 6.36 & $0.34(1)$ & $(70)$ \\
\hline 1,3,6,9-TeCDD & -0.95 & 5.29 & $0.21(1)$ & $(70)$ \\
\hline 1,3,7,9-TeCDD & -0.95 & 5.29 & $0.16(0.16,2)$ & $(70,71)$ \\
\hline $1,4,6,8-\mathrm{TeCDF}$ & -0.84 & 6.63 & $0.1(1)$ & $(70)$ \\
\hline $2,3,4,6,7,8-\mathrm{HxCDF}$ & -1.28 & 7.92 & $0.77(0.32,7)$ & $(67-70,72)$ \\
\hline 2,3,4,6,7-PeCDF & -0.91 & 7.27 & $0.17(1)$ & $(70)$ \\
\hline 2,3,4,7,8-PeCDF & -1.01 & 7.27 & $0.91(0.52,8)$ & $(67-72)$ \\
\hline $2,3,6,7-\mathrm{TeCDF}$ & -0.82 & 6.27 & $0.08(1)$ & $(70)$ \\
\hline 2,3,7,8-TeCDD & -2.05 & 6.8 & $1.5(0.96,6)$ & $(67-69,71,72)$ \\
\hline $2,3,7,8-\mathrm{TeCDF}$ & -1.05 & 6.63 & $1.15(0.88,7)$ & $(68-72)$ \\
\hline $2,4,6,8-\mathrm{TeCDF}$ & -0.86 & 6.36 & $0.06(1)$ & $(70)$ \\
\hline OCDD & -0.82 & 8.2 & $0.22(0.24,6)$ & $(69-72)$ \\
\hline OCDF & -1.64 & 8.6 & $0.49(0.67,7)$ & $(68-72)$ \\
\hline \multicolumn{5}{|c|}{ Polychlorinated Biphenyls } \\
\hline PCB-1 & -1.21 & 4.46 & $0.61(1)$ & $(73)$ \\
\hline PCB-2 & -1.19 & 4.69 & $0.4(1)$ & (73) \\
\hline PCB-3 & -1.2 & 4.69 & $1.03(0.81,2)$ & $(43,73)$ \\
\hline PCB-4 & -1.94 & 4.65 & $1.64(0.93,2)$ & $(43,73)$ \\
\hline PCB-5 & -2.31 & 4.97 & $1.6(1.7,2)$ & $(43,73)$ \\
\hline PCB-6 & -2.7 & 5.06 & $2.36(2.47,2)$ & $(43,73)$ \\
\hline PCB-7 & -1.39 & 5.07 & $2.01(1.97,2)$ & $(43,73)$ \\
\hline PCB-8 & -1.97 & 5.07 & $1.6(1.11,4)$ & $(43,56,73,74)$ \\
\hline PCB-9 & -2.54 & 5.06 & $1.31(0.91,2)$ & (73) \\
\hline PCB-10 & -1.33 & 4.84 & $0.64(1)$ & (73) \\
\hline PCB-11 & -0.37 & 5.28 & $0.35(1)$ & (73) \\
\hline PCB-15 & -2.01 & 5.3 & $0.5(1)$ & (73) \\
\hline PCB-16 & -2.71 & 5.16 & $1.18(0.36,2)$ & $(57,73)$ \\
\hline PCB-17 & -2.2 & 5.25 & $1.55(0.66,3)$ & $(60,73,75)$ \\
\hline
\end{tabular}




\begin{tabular}{|c|c|c|c|c|}
\hline PCB-18 & -2.61 & 5.24 & $1.29(0.52,10)$ & $\begin{array}{l}(17,23,43,53,56,57,60, \\
73-75)\end{array}$ \\
\hline PCB-19 & -2.67 & 5.02 & $1.8(1.42,4)$ & $(57,60,73,75)$ \\
\hline PCB-21 & -1.63 & 5.51 & $1.35(1)$ & $(23)$ \\
\hline PCB-22 & -2.3 & 5.58 & $1.41(0.55,5)$ & $(23,57,60,73,75)$ \\
\hline PCB-25 & -2.91 & 5.67 & $1.41(0.77,3)$ & $(57,73,75)$ \\
\hline PCB-26 & -3.02 & 5.66 & $1.75(0.82,3)$ & $(60,73,75)$ \\
\hline PCB-27 & -2.18 & 5.44 & $1.28(0.76,2)$ & $(73,75)$ \\
\hline PCB-28 & -2.04 & 5.67 & $2.3(1.88,19)$ & $\begin{array}{l}(4,11,15,17,18,36,37 \\
40,43,46,56,57,73-76)\end{array}$ \\
\hline PCB-31 & -2.07 & 5.67 & $1.78(1.05,5)$ & $(37,40,43,73,75)$ \\
\hline PCB-32 & -2.6 & 5.44 & $1.53(1.03,2)$ & $(73,75)$ \\
\hline PCB-35 & -2.18 & 5.82 & $1.38(1)$ & $(23)$ \\
\hline PCB-36 & -2.21 & 5.88 & $0.86(1)$ & (73) \\
\hline PCB-37 & -2.24 & 5.83 & $1.2(0.71,3)$ & $(60,73,75)$ \\
\hline PCB-40 & -2.13 & 5.66 & $0.72(1)$ & (73) \\
\hline PCB-41 & -2.33 & 5.69 & $3.37(1)$ & $(60)$ \\
\hline PCB-42 & -3.11 & 5.76 & $2.23(1.65,2)$ & $(60,73)$ \\
\hline PCB-44 & -2.09 & 5.75 & $1.97(0.9,7)$ & $(17,56,57,60,73-75)$ \\
\hline PCB-45 & -2.8 & 5.53 & $1.9(1.53,2)$ & $(73,75)$ \\
\hline PCB-46 & -2.5 & 5.53 & $0.77(1)$ & $(73)$ \\
\hline PCB-47 & -2.38 & 5.85 & $5.2(2.3,2)$ & $(37,75)$ \\
\hline PCB-48 & -2.4 & 5.78 & $2.65(1)$ & $(75)$ \\
\hline PCB-49 & -2.36 & 5.85 & $1.87(0.98,4)$ & $(43,56,73,75)$ \\
\hline PCB-51 & -2.81 & 5.63 & $0.88(1)$ & $(73)$ \\
\hline PCB-52 & -3.14 & 5.84 & $2.12(1.34,36)$ & $\begin{array}{l}(11,14,15,18,23,35,36 \\
39,40,43,46,56,57,60, \\
74-76)\end{array}$ \\
\hline PCB-53 & -3.11 & 5.62 & $1.38(0.76,2)$ & $(73,75)$ \\
\hline PCB-55 & -2.4 & 6.11 & $0.9(1)$ & $(73)$ \\
\hline
\end{tabular}




\begin{tabular}{|c|c|c|c|c|}
\hline PCB-56 & -2.63 & 6.11 & $1.71(1.13,3)$ & $(57,73,75)$ \\
\hline PCB-58 & -2.35 & 6.17 & $0.84(1)$ & (73) \\
\hline PCB-59 & -2.88 & 5.95 & $0.97(1)$ & (73) \\
\hline PCB-60 & -2.25 & 6.11 & $3.12(1.81,3)$ & $(60,73,75)$ \\
\hline PCB-63 & -2.44 & 6.17 & $1.17(1)$ & (73) \\
\hline PCB-64 & -2.59 & 5.95 & $1.8(0.92,5)$ & $(4,23,43,73,75)$ \\
\hline PCB-66 & -2.39 & 6.2 & $2.32(0.99,7)$ & $(23,43,56,57,73-75)$ \\
\hline PCB-67 & -2.4 & 6.2 & $0.92(0.17,2)$ & $(57,73)$ \\
\hline PCB-68 & -2.36 & 6.04 & $1.24(1)$ & (73) \\
\hline PCB-70 & -2.91 & 6.2 & $2.39(1.42,5)$ & $(23,43,60,73,75)$ \\
\hline PCB-71 & -2.4 & 5.98 & $1.9(0.62,2)$ & $(57,60)$ \\
\hline PCB-74 & -2.9 & 6.2 & $2.96(1.61,8)$ & $\begin{array}{l}(4,23,43,56,57,60,73 \\
75)\end{array}$ \\
\hline PCB-77 & -1.52 & 6.36 & $1.72(1.19,8)$ & $(69-71,73-75,77)$ \\
\hline PCB-81 & -1.85 & 6.36 & $2.68(2.83,5)$ & $(69-71,77)$ \\
\hline PCB-81/87/115 & -2.67 & 6.46 & $3.71(1)$ & $(60)$ \\
\hline PCB-82 & -2.62 & 6.2 & $2.42(1.48,3)$ & $(57,73,75)$ \\
\hline PCB-83 & -2.67 & 6.26 & $2.03(1.29,2)$ & $(60,73)$ \\
\hline PCB-84 & -1.76 & 6.04 & $2.98(0.39,2)$ & $(60,75)$ \\
\hline PCB-85 & -2.51 & 6.3 & $1.24(1)$ & (73) \\
\hline PCB-87 & -2.4 & 6.29 & $3.36(1.85,4)$ & $(43,56,57,75)$ \\
\hline PCB-91 & -2.81 & 6.13 & $2.05(1.35,3)$ & $(73,75,78)$ \\
\hline PCB-92 & -2.56 & 6.35 & $3.2(1.7,3)$ & $(60,73,75)$ \\
\hline PCB-94 & -2.62 & 6.13 & $1.02(1)$ & (73) \\
\hline PCB-95 & -2.92 & 6.13 & $2.25(0.75,4)$ & $(42,43,75,78)$ \\
\hline PCB-96 & -2.62 & 5.71 & $0.8(1)$ & (73) \\
\hline PCB-97 & -2.9 & 6.29 & $2.67(1.01,3)$ & $(4,43,75)$ \\
\hline PCB-99 & -3.05 & 6.39 & $4.39(3.69,28)$ & $\begin{array}{l}(4,14,23,36,37,43,53 \\
56,57,60,73,75)\end{array}$ \\
\hline
\end{tabular}




\begin{tabular}{|c|c|c|c|c|}
\hline PCB-100 & -2.62 & 6.23 & $3.2(2.65,2)$ & $(73,75)$ \\
\hline PCB-101 & -2.57 & 6.38 & $2.8(1.53,35)$ & $\begin{array}{l}(4,11,14,15,18,23,35 \\
36,40,42,46,56,60,74- \\
76)\end{array}$ \\
\hline PCB-103 & -3.51 & 6.22 & $1.49(1)$ & (73) \\
\hline PCB-105 & -1.96 & 6.65 & $4.12(2.96,22)$ & $\begin{array}{l}(4,17,23,35-37,40,43 \\
46,56,60,70,71,73-77 \\
79)\end{array}$ \\
\hline PCB-109 & -2.62 & 6.48 & $1.28(1)$ & (73) \\
\hline PCB-110 & -2.98 & 6.48 & $2.65(0.75,8)$ & $\begin{array}{l}(4,23,42,43,56,57,60 \\
75)\end{array}$ \\
\hline PCB-113 & -2.62 & 6.54 & $1.98(1)$ & (23) \\
\hline PCB-114 & -2.59 & 6.65 & $8.11(6.94,4)$ & $(70,71,73,77)$ \\
\hline PCB-118 & -2.18 & 6.74 & $6.59(8.72,30)$ & $\begin{array}{l}(4,15,17,18,23,35-37 \\
40,43,46,47,53,56,60 \\
70,71,73-77,79,80)\end{array}$ \\
\hline PCB-122 & -2.62 & 6.64 & $0.55(1)$ & $(73)$ \\
\hline PCB-123 & -2.62 & 6.74 & $6.12(5.41,4)$ & $(35,70,71,73)$ \\
\hline PCB-124 & -2.62 & 6.73 & $2.92(2.76,2)$ & $(73,75)$ \\
\hline PCB-126 & -2.56 & 6.89 & $3.45(2.46,7)$ & $(69-71,73,74,77)$ \\
\hline PCB-128 & -2.34 & 6.74 & $8.72(15.12,12)$ & $\begin{array}{l}(4,17,23,47,55,60,73- \\
75,79)\end{array}$ \\
\hline PCB-129 & -2.75 & 6.73 & $1.21(1)$ & (73) \\
\hline PCB-130 & -2.77 & 6.8 & $3.27(2.44,2)$ & $(73,75)$ \\
\hline PCB-131 & -2.66 & 6.58 & $2.12(1.65,2)$ & $(60,73)$ \\
\hline PCB-132 & -2.17 & 6.58 & $2.03(1.09,2)$ & $(43,73)$ \\
\hline PCB-133 & -2.67 & 6.86 & $1.32(1)$ & (73) \\
\hline PCB-134 & -2.73 & 6.55 & $2.8(1.52,3)$ & $(60,73,75)$ \\
\hline PCB-135 & -2.54 & 6.64 & $3.13(1.68,3)$ & $(60,73,75)$ \\
\hline PCB-136 & -2.47 & 6.22 & $2.42(1.14,5)$ & $(43,60,73,75,78)$ \\
\hline PCB-137 & -2.7 & 6.83 & $3.61(3.22,2)$ & $(73,75)$ \\
\hline PCB-138 & -2.54 & 6.83 & $5.99(8.58,46)$ & $\begin{array}{l}(4,11,14,15,18,23,35- \\
37,40,42,43,46-48,57\end{array}$ \\
\hline
\end{tabular}


$60,63,73-76,79,80)$

\begin{tabular}{|c|c|c|c|c|}
\hline PCB-139 & -3.58 & 6.67 & $2.18(1)$ & (23) \\
\hline PCB-141 & -2.71 & 6.82 & $3.75(2.07,4)$ & $(4,60,73,75)$ \\
\hline PCB-144 & -2.48 & 6.67 & $2.89(1.97,2)$ & $(73,75)$ \\
\hline PCB-146 & -2.57 & 6.89 & $2.73(1.39,5)$ & $(42,56,57,73,75)$ \\
\hline PCB-147 & -2.84 & 6.64 & $3.11(0.94,2)$ & $(57,60)$ \\
\hline PCB-149 & -2.73 & 6.67 & $2.98(1.6,11)$ & $\begin{array}{l}(4,15,37,42,43,56,73, \\
75,78)\end{array}$ \\
\hline PCB-151 & -2.84 & 6.64 & $2.77(2.01,4)$ & $(43,56,73,75)$ \\
\hline PCB-153 & -3.31 & 6.92 & $6(6.74,50)$ & $\begin{array}{l}(4,11,14,15,18,26,29, \\
31,34-37,40,42,43,46- \\
48,50,56,57,73,74,76, \\
78-80)\end{array}$ \\
\hline PCB-154 & -2.84 & 6.76 & $1.34(1)$ & $(73)$ \\
\hline PCB-156 & -2.58 & 7.18 & $5.15(5.69,14)$ & $\begin{array}{l}(4,35,40,43,47,56,70, \\
71,73,75,77,79)\end{array}$ \\
\hline PCB-157 & -2.83 & 7.18 & $5.85(6.86,6)$ & $(56,60,70,71,73,75)$ \\
\hline PCB-158 & -2.37 & 7.02 & $4.38(4.27,2)$ & $(4,73)$ \\
\hline PCB-163 & -2.6 & 6.99 & $4.39(1)$ & $(75)$ \\
\hline PCB-164 & -2.84 & 7.02 & $4.63(1)$ & $(75)$ \\
\hline PCB-167 & -2.42 & 7.27 & $7.92(9.19,4)$ & $(70,71,73,75)$ \\
\hline PCB-169 & -2.05 & 7.42 & $4.44(4.44,5)$ & $(69-71,77)$ \\
\hline PCB-170 & -3.06 & 7.27 & $6.81(4.69,9)$ & $(35,47,56,73-75,79)$ \\
\hline PCB-171 & -2.54 & 7.11 & $3.24(2.69,2)$ & $(73,75)$ \\
\hline PCB-172 & -2.58 & 7.33 & $3.33(1.72,3)$ & $(56,60,73)$ \\
\hline PCB-173 & -3.06 & 7.02 & $1.85(1)$ & $(57)$ \\
\hline PCB-174 & -2.5 & 7.11 & $2.65(1.14,6)$ & $(42,43,57,73,75,78)$ \\
\hline PCB-175 & -3.06 & 7.17 & $1.08(1)$ & (73) \\
\hline PCB-176 & -3.06 & 6.76 & $0.93(0.32,2)$ & $(73,78)$ \\
\hline PCB-177 & -2.47 & 7.08 & $2.87(1.16,6)$ & $(43,56,57,60,73,75)$ \\
\hline
\end{tabular}




\begin{tabular}{|c|c|c|c|c|}
\hline PCB-178 & -3.06 & 7.14 & $3.72(2.48,4)$ & $(4,56,73,75)$ \\
\hline PCB-179 & -3.06 & 6.73 & $1.77(0.59,2)$ & $(57,73)$ \\
\hline PCB-180 & -3.06 & 7.38 & $5.2(3.44,43)$ & $\begin{array}{l}(4,11,14,17,18,26,29 \\
35,36,40,43,46,47,53 \\
55-57,73,74,76,79,80)\end{array}$ \\
\hline PCB-183 & -2.55 & 7.2 & $3.46(2.08,8)$ & $\begin{array}{l}(4,43,47,56,60,73,75 \\
78)\end{array}$ \\
\hline PCB-185 & -2.52 & 7.11 & $1.29(1)$ & $(73)$ \\
\hline PCB-187 & -2.5 & 7.17 & $4.37(2.17,12)$ & $\begin{array}{l}(4,42,43,55-57,60,74 \\
75,79)\end{array}$ \\
\hline PCB-189 & -2.0 & 7.71 & $5.99(5.1,4)$ & $(56,70,71,73)$ \\
\hline PCB-190 & -3.06 & 7.46 & $1.37(1)$ & $(73)$ \\
\hline PCB-191 & -3.06 & 7.55 & $1.12(1)$ & $(73)$ \\
\hline PCB-193 & -3.06 & 7.52 & $1.7(1)$ & $(73)$ \\
\hline PCB-194 & -2.57 & 7.8 & $6.26(6.1,9)$ & $(43,47,53,56,57,73,79)$ \\
\hline PCB-195 & -2.04 & 7.56 & $3.27(1.75,5)$ & $(11,56,57,73,74)$ \\
\hline PCB-196 & -3.28 & 7.65 & $1.27(1)$ & (73) \\
\hline PCB-198 & -3.28 & 7.62 & $1.55(1)$ & $(73)$ \\
\hline PCB-199 & -2.52 & 7.2 & $3.59(2.52,5)$ & $(47,56,57,60,73)$ \\
\hline PCB-200 & -3.28 & 7.27 & $0.7(1)$ & (73) \\
\hline PCB-201 & -2.49 & 7.62 & $1.6(0.86,2)$ & $(43,73)$ \\
\hline PCB-202 & -2.92 & 7.24 & $1.09(1)$ & $(73)$ \\
\hline PCB-203 & -3.28 & 7.65 & $1.66(0.39,2)$ & $(57,73)$ \\
\hline PCB-204 & -3.28 & 7.3 & $1.8(1)$ & (43) \\
\hline PCB-206 & -2.15 & 8.09 & $4.98(2.05,6)$ & $(11,17,55-57,74)$ \\
\hline PCB-207 & -2.5 & 7.74 & $3.61(1)$ & $(60)$ \\
\hline PCB-208 & -2.41 & 7.71 & $3.21(1)$ & $(60)$ \\
\hline PCB-209 & -2.33 & 8.18 & $3.56(1.52,4)$ & $(11,53,56,60)$ \\
\hline \multicolumn{5}{|c|}{ olychlorinated Naphthalenes } \\
\hline PCN-19 & -1.34 & 5.1 & $0.85(1)$ & $(77)$ \\
\hline
\end{tabular}




\begin{tabular}{|c|c|c|c|c|}
\hline $\mathrm{PCN}-24$ & -1.34 & 5.1 & $0.69(1)$ & (77) \\
\hline PCN-27/30 & -1.36 & 5.75 & $0.52(1)$ & (77) \\
\hline PCN-35 & -1.56 & 5.75 & $0.71(0.28,2)$ & $(35,77)$ \\
\hline PCN-37 & -1.56 & 5.75 & $0.45(1)$ & $(35)$ \\
\hline PCN-39 & -1.56 & 5.75 & $0.47(0.15,2)$ & $(35,77)$ \\
\hline PCN-40 & -1.56 & 5.75 & $0.28(1)$ & $(35)$ \\
\hline PCN-41 & -1.56 & 5.75 & $0.24(1)$ & $(35)$ \\
\hline PCN-42 & -1.7 & 6.19 & $1.21(1.16,2)$ & $(35,77)$ \\
\hline $\mathrm{PCN}-43$ & -1.56 & 5.76 & $0.36(1)$ & $(35)$ \\
\hline PCN-44 & -1.56 & 5.75 & $1.05(1)$ & (77) \\
\hline PCN-45 & -1.7 & 6.19 & $0.31(1)$ & (35) \\
\hline PCN-46 & -1.56 & 5.75 & $0.3(1)$ & $(35)$ \\
\hline PCN-47 & -1.58 & 5.81 & $0.99(0.45,2)$ & $(35,77)$ \\
\hline PCN-48 & -1.56 & 5.75 & $0.45(1)$ & $(35)$ \\
\hline $\mathrm{PCN}-50$ & -1.97 & 7 & $0.83(0.4,2)$ & $(35,77)$ \\
\hline PCN-51 & -1.78 & 6.39 & $0.91(0.22,2)$ & $(35,77)$ \\
\hline PCN-52 & -1.78 & 6.39 & $2.13(1)$ & $(77)$ \\
\hline PCN-52/60 & -1.78 & 6.39 & $2.69(1)$ & $(77)$ \\
\hline PCN-53 & -1.91 & 6.8 & $1.15(1)$ & (77) \\
\hline PCN-54 & -1.78 & 6.39 & $1.99(0.45,2)$ & $(35,77)$ \\
\hline PCN-55 & -1.78 & 6.39 & $1.03(1)$ & $(35)$ \\
\hline PCN-57 & -1.78 & 6.39 & $0.72(0.17,2)$ & $(35,77)$ \\
\hline PCN-58 & -1.78 & 6.39 & $1.4(1.1,2)$ & $(35,77)$ \\
\hline PCN-59 & -1.78 & 6.39 & $0.94(1)$ & (77) \\
\hline PCN-60 & -1.78 & 6.39 & $1.48(0.9,2)$ & $(35,77)$ \\
\hline PCN-61 & -1.78 & 6.39 & $2.07(0.57,2)$ & $(35,77)$ \\
\hline PCN-62 & -1.78 & 6.39 & $0.77(0.37,2)$ & $(35,77)$ \\
\hline PCN-64 & -2.0 & 7.04 & $1.62(1)$ & (77) \\
\hline
\end{tabular}




$\begin{array}{lllll}\text { PCN-65 } & -2.0 & 7.04 & 1.49(1) & (77) \\ \text { PCN-66 } & -2.21 & 7.7 & 3.37(1) & (77) \\ \text { PCN-67 } & -2.0 & 7.04 & 3.29(1) & (77) \\ \text { PCN-68 } & -2.14 & 7.5 & 1.7(1) & (77) \\ \text { PCN-69 } & -2.14 & 7.5 & 2.37(1) & (77) \\ \text { PCN-73 } & -2.38 & 8.2 & 2.32(1) & (77) \\ \text { PCN-74 } & -2.22 & 7.68 & 1.16(1) & (77) \\ \text { PCN-75 } & -1.28 & 8.5 & 0.69(1) & (77)\end{array}$

Polycyclic Aromatic Hydrocarbons

\begin{tabular}{|c|c|c|c|c|}
\hline 1-Methylphenanthrene & -0.45 & 5.08 & $0.29(0.01,2)$ & $(17,35)$ \\
\hline 1-Methylpyrene & 0.12 & 5.48 & $0.19(1)$ & $(35)$ \\
\hline 2-Methylphenanthren & -0.38 & 5.15 & $0.44(1)$ & $(81)$ \\
\hline 2-Methylpyrene & 0.12 & 5.48 & $0.24(1)$ & $(35)$ \\
\hline 3-Methylphenanthrene & -0.38 & 5.15 & $0.2(0.12,2)$ & $(17)$ \\
\hline 9H-Fluorene & -0.89 & 4.18 & $1.22(0.09,2)$ & $(33,82)$ \\
\hline 9-Methylphenanthrene & -0.39 & 4.89 & $0.23(1)$ & $(17)$ \\
\hline Acenaphthylene & -0.98 & 3.94 & $0.73(0.39,2)$ & $(33,82)$ \\
\hline Acenapthene & 0.44 & 4.15 & $1.19(0.23,2)$ & $(33,82)$ \\
\hline Anthracene & -0.66 & 4.45 & $0.71(0.56,3)$ & $(17,33,82)$ \\
\hline Benzo[e]acephenanthrylene & -0.59 & 5.78 & $0.69(0.73,2)$ & $(17,33)$ \\
\hline Benzo[e]pyrene & -0.21 & 6.44 & $0.33(0.24,3)$ & $(17,35,82)$ \\
\hline Benzo[ghi]fluoranthene & -0.04 & 5.52 & $0.39(1)$ & $(35)$ \\
\hline Benzo[ghi]perylene & 0.37 & 6.7 & $0.62(0.35,4)$ & $(17,33,35,82)$ \\
\hline Benzo[k]fluoranthene & -0.1 & 6.11 & $0.55(1)$ & $(35)$ \\
\hline benzo[k]tetraphene & -0.71 & 6.75 & $1.14(0.4,2)$ & $(33,82)$ \\
\hline Benzo[pqr]tetraphene & -0.2 & 5.99 & $0.59(0.49,5)$ & $(17,18,33,35,82)$ \\
\hline Chrysene & -0.66 & 5.81 & $0.46(0.43,4)$ & $(17,33,35,82)$ \\
\hline Coronene & 0.7 & 6.49 & $0.11(1)$ & $(35)$ \\
\hline
\end{tabular}




$\begin{array}{lllll}\text { Cyclopenta[cd]pyrene } & -0.41 & 5.7 & 0.25(1) & (17) \\ \text { Fluoranthene } & -0.02 & 5.16 & 0.45(0.51,4) & (17,33,35,82) \\ \text { Indeno[1,2,3-cd]pyrene } & -0.23 & 6.76 & 0.89(0.7,4) & (17,33,35,82) \\ \text { Naphthalene } & -0.82 & 3.3 & 0.5(1) & (82) \\ \text { Perylene } & -0.1 & 6.25 & 0.3(0.09,3) & (17,35,82) \\ \text { Phenanthrene } & -0.81 & 4.46 & 0.65(0.42,4) & (17,33,35,82) \\ \text { Pyrene } & -0.81 & 4.88 & 0.41(0.41,5) & (17,18,33,35,82) \\ \text { Tetraphene } & -0.64 & 5.76 & 0.55(0.38,4) & (17,33,35,82)\end{array}$


Table S2. Regression statistics of $\log _{10}$ TMF vs. latitude for "cold" food webs (containing only ectotherms)

\begin{tabular}{|c|c|c|c|c|c|c|c|c|}
\hline \multirow[b]{2}{*}{ Compound } & \multicolumn{4}{|c|}{ Marine + Freshwater } & \multicolumn{4}{|c|}{ Freshwater only } \\
\hline & $\mathrm{n}$ & $\mathrm{r}^{2}$ & $\mathrm{~F}$ & $\mathrm{p}$ & $\mathrm{n}$ & $\mathrm{r}^{2}$ & $\mathrm{~F}$ & $\mathrm{p}$ \\
\hline alpha-HCH & 22 & 0.26 & 6.85 & 0.017 & 21 & 0.35 & 10.43 & 0.004 \\
\hline gamma-HCH (lindane) & 22 & $<0.01$ & $<0.01$ & 0.993 & 21 & $<0.01$ & 0.01 & 0.939 \\
\hline trans-nonachlor & 22 & 0.08 & 1.82 & 0.193 & 21 & 0.08 & 1.60 & 0.222 \\
\hline dieldrin & 20 & 0.04 & 0.67 & 0.425 & 19 & 0.05 & 0.86 & 0.368 \\
\hline alpha-chlordane & 21 & 0.15 & 3.37 & 0.082 & 20 & 0.14 & 2.82 & 0.110 \\
\hline sum-DDT & 9 & 0.16 & 1.33 & 0.287 & 8 & 0.58 & 8.22 & 0.029 \\
\hline sum-HCH & 8 & 0.39 & 3.78 & 0.100 & 7 & 0.67 & 9.92 & 0.025 \\
\hline sum-PBDE & 10 & 0.01 & 0.09 & 0.774 & N/A & N/A & N/A & N/A \\
\hline sum-PCB & 20 & 0.00 & 0.06 & 0.808 & 17 & 0.03 & 0.41 & 0.533 \\
\hline HCB & 26 & 0.02 & 0.59 & 0.451 & 24 & 0.05 & 1.10 & 0.306 \\
\hline PBDE-100 & 10 & 0.14 & 1.33 & 0.283 & 7 & 0.70 & 11.89 & $0.018 *$ \\
\hline PBDE-153 & 8 & 0.02 & 0.10 & 0.758 & N/A & N/A & N/A & N/A \\
\hline PBDE-154 & 7 & 0.51 & 5.24 & 0.071 & N/A & N/A & N/A & N/A \\
\hline PBDE-28 & 8 & 0.02 & 0.15 & 0.716 & N/A & N/A & N/A & N/A \\
\hline PBDE-47 & 14 & 0.16 & 2.36 & 0.151 & 10 & 0.18 & 1.81 & 0.216 \\
\hline PBDE-99 & 14 & 0.06 & 0.73 & 0.410 & 11 & 0.05 & 0.50 & 0.497 \\
\hline PCB-101 & 28 & 0.02 & 0.49 & 0.490 & 26 & 0.03 & 0.82 & 0.373 \\
\hline PCB-105 & 14 & 0.12 & 1.58 & 0.233 & 9 & 0.59 & 10.02 & $0.016 *$ \\
\hline PCB-110 & 7 & 0.05 & 0.24 & 0.644 & N/A & N/A & N/A & N/A \\
\hline PCB-118 & 17 & 0.06 & 1.01 & 0.331 & 11 & 0.68 & 19.52 & $0.002 *$ \\
\hline PCB-128 & 8 & 0.01 & 0.08 & 0.798 & N/A & N/A & N/A & N/A \\
\hline PCB-138 & 32 & $<0.01$ & 0.01 & 0.916 & 29 & 0.01 & 0.14 & 0.707 \\
\hline PCB-153 & 35 & 0.03 & 0.89 & 0.352 & 32 & 0.02 & 0.58 & 0.451 \\
\hline PCB-156 & 8 & 0.15 & 1.07 & 0.341 & N/A & N/A & N/A & N/A \\
\hline PCB-18 & 10 & 0.10 & 0.90 & 0.371 & N/A & N/A & N/A & N/A \\
\hline PCB-180 & 34 & 0.00 & 0.06 & 0.813 & 28 & 0.00 & 0.03 & 0.863 \\
\hline PCB-183 & 7 & 0.39 & 3.18 & 0.134 & N/A & N/A & N/A & N/A \\
\hline PCB-187 & 8 & 0.07 & 0.48 & 0.517 & N/A & N/A & N/A & N/A \\
\hline PCB-28 & 11 & 0.00 & 0.02 & 0.893 & 8 & 0.14 & 0.94 & 0.370 \\
\hline PCB-44 & 7 & 0.05 & 0.23 & 0.649 & N/A & N/A & N/A & N/A \\
\hline PCB-52 & 30 & 0.01 & 0.14 & 0.713 & 28 & 0.04 & 1.10 & 0.304 \\
\hline PCB-66 & 7 & 0.02 & 0.09 & 0.778 & N/A & N/A & N/A & N/A \\
\hline PCB-74 & 7 & 0.16 & 0.93 & 0.378 & N/A & N/A & N/A & N/A \\
\hline PCB-99 & 25 & 0.04 & 0.88 & 0.359 & 22 & 0.004 & 0.08 & 0.775 \\
\hline $\mathrm{p}, \mathrm{p}^{\prime}-\mathrm{DDE}$ & 31 & 0.05 & 1.62 & 0.213 & 29 & 0.08 & 2.28 & 0.142 \\
\hline
\end{tabular}

*indicates coefficient was negative (i.e. higher TMFs at lower latitudes) 
Figure S1. Quantile regression ( $\tau \in\{0.05,0.10,0.25,0.50,0.75,0.90,0.95\}$, numbered in plot) smoothing spline models for the global dataset $(n=1,591)$ for $\log _{10}$ TMF as a function of $\log _{10}$ $K_{\mathrm{OW}}$. The number and location of knots in $\log _{10} K_{\mathrm{Ow}}$ were allowed to vary among quantiles to optimize the minimization criteria using a smoothing parameter $\lambda=1(83)$. Different color lines correspond to the quantiles. Note the near linear decreasing function of $\log _{10}$ TMF for $\log _{10} K_{\mathrm{OW}}$ $<5$, with a near linear increase in $\log _{10}$ TMF up to $\log _{10} K_{\mathrm{OW}}=7$, followed by near linear decrease for $\log _{10} K_{\mathrm{OW}}>7$. These estimates suggested that a simpler piecewise linear function with common breakpoints (knots) around $\log _{10} K_{\mathrm{OW}}=5$ and 7 for all quantiles would be reasonable.

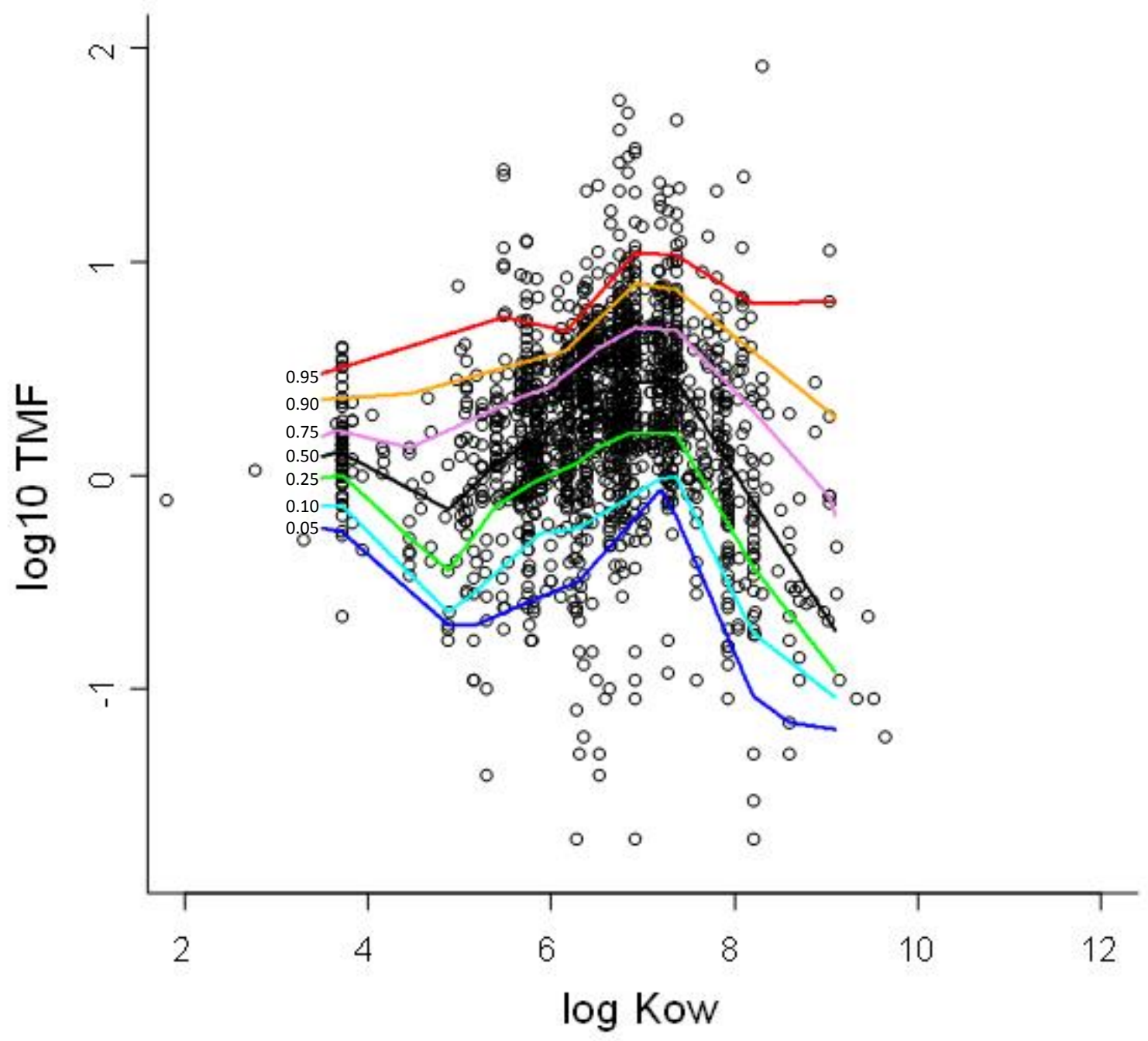


Figure S2. Quantile regression ( $\tau \in\{0.05,0.10,0.25,0.50,0.75,0.90,0.95\})$ estimates (points) for model (1) for the global dataset $(n=1,591)$ with linear b-splines (knots at 4.9 and 7.2) for $\log _{10} K_{\text {OW }}$ and their $95 \%$ confidence intervals (shaded polygons) based on a weightedexponential bootstrapping procedure that accounts for heterogeneity with 1,000 resamples. Minimum (min) and maximum (max) values of $\log _{10} K_{\mathrm{OW}}$ used as boundary knots by the bsplines were 1.8 and 9.64, respectively.
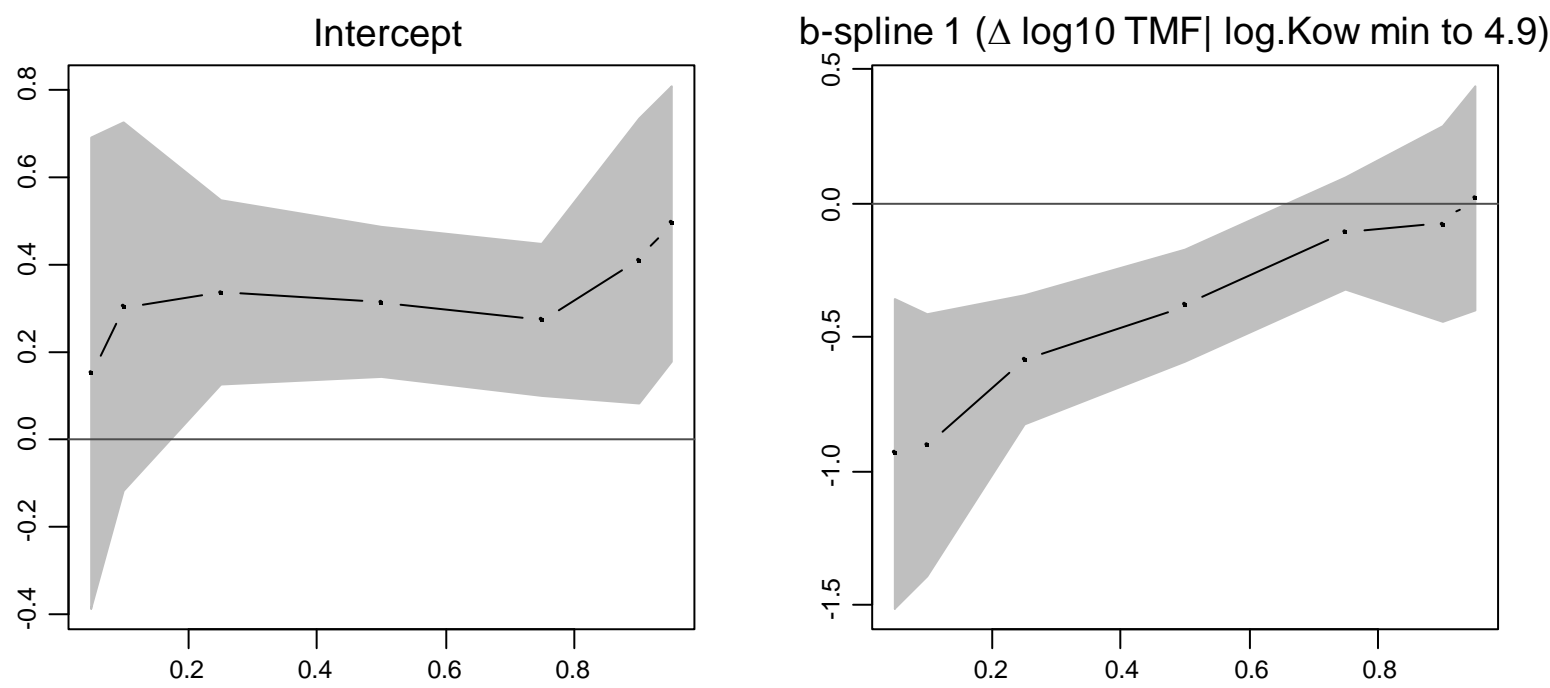

b-spline $2(\Delta \log 10 \mathrm{TMF} \mid \log$. Kow $\min$ to 7.2$)$

b-spline $3(\Delta \log 10 \mathrm{TMF} \mid \log$. Kow min to max $)$
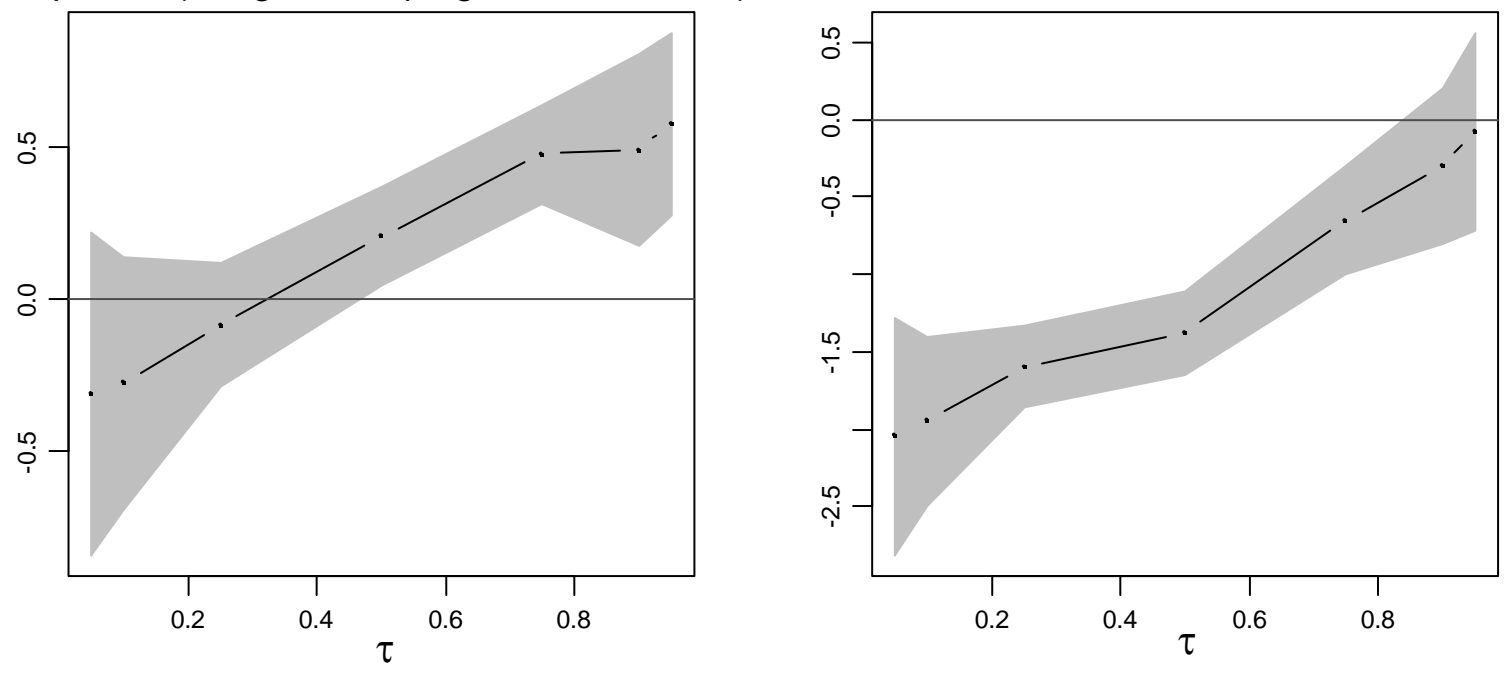
Figure S3. Quantile regression $(\tau \in\{0.05,0.10,0.25,0.50,0.75,0.90,0.95\} . v)$ estimates (points) for model (2) for the global dataset $(n=1,591)$ with a linear function of $\log _{10} k_{\mathrm{M}}(n=$ 1,533 ) and their $95 \%$ confidence intervals (shaded polygons) based on a weighted-exponential bootstrapping procedure that accounts for heterogeneity with 1,000 resamples.
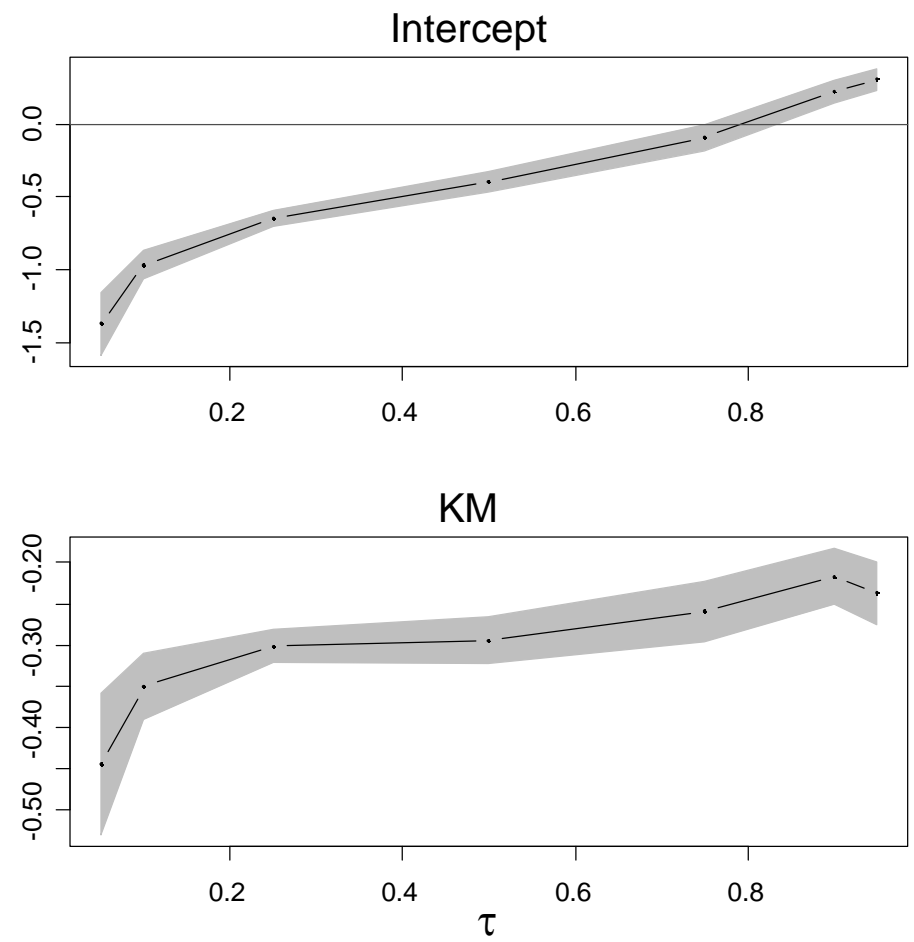
Figure S4. Quantile regression ( $\tau \in\{0.05,0.10,0.25,0.50,0.75,0.90,0.95\})$ estimates (points) for model (3) for the global dataset $(n=1,591)$ with linear b-splines (knots at 4.9 and 7.2) for $\log _{10} K_{\mathrm{OW}}$ and a linear function of $\log _{10} k_{\mathrm{M}}(n=1,533)$ and their $95 \%$ confidence intervals (shaded polygons) based on a weighted-exponential bootstrapping procedure that accounts for heterogeneity with 1,000 resamples. Minimum (min) and maximum (max) values of $\log _{10} K_{\mathrm{OW}}$ used as boundary knots by the b-splines were 1.8 and 9.64 , respectively.
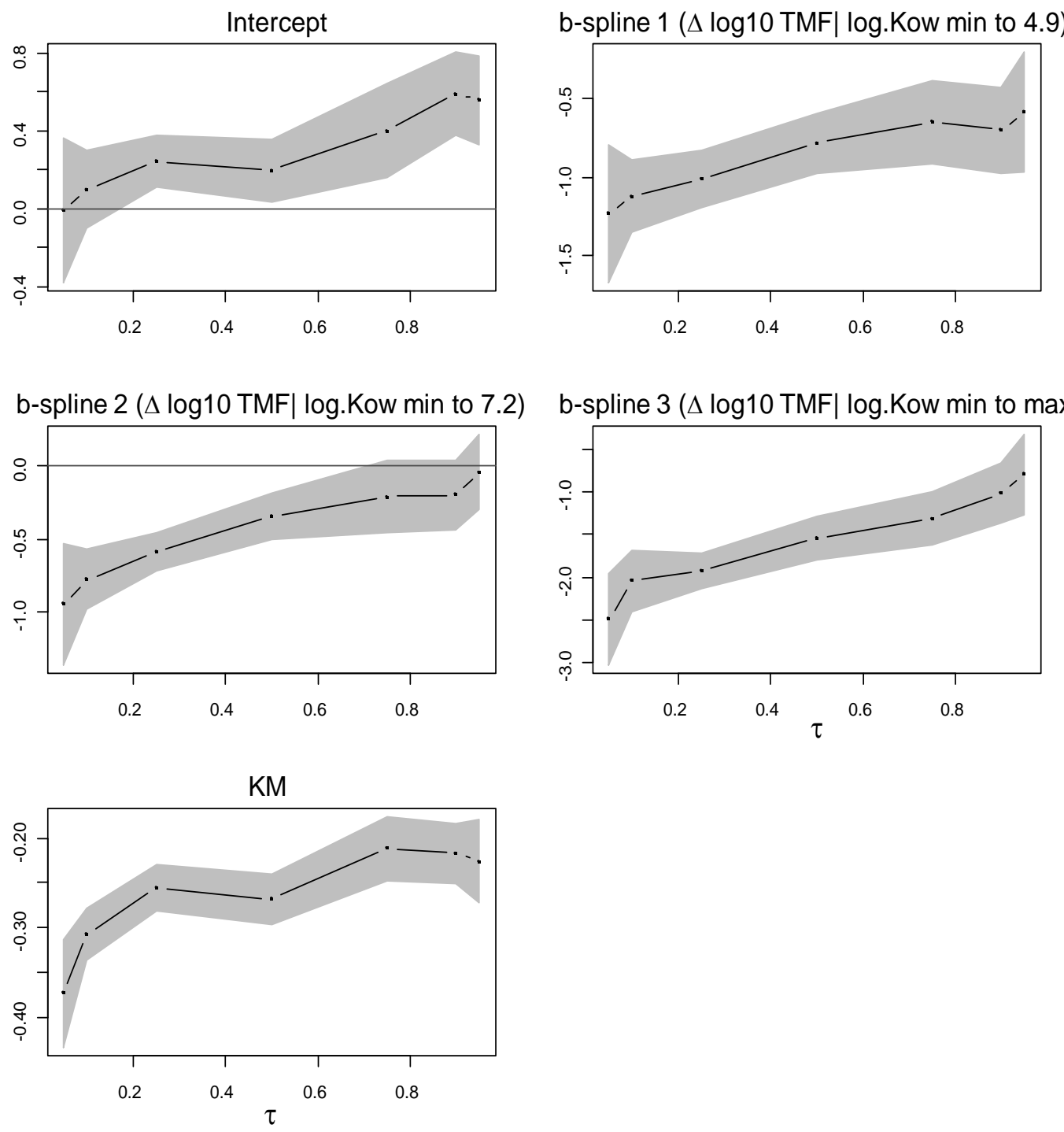
Figure S5. (A) Differences in Akaike Information Criteria ( $\triangle \mathrm{AIC})$ between a null quantile regression $(\tau \in\{0.05,0.10,0.25,0.50,0.75,0.90,0.95\})$ model with just an intercept for $\log _{10}$ TMF and (1) the model with linear b-splines (knots at 4.9 and 7.2) for $\log _{10} K_{\text {OW }}$ (black lines); (2) the model linear in $\log _{10} k_{\mathrm{M}}$ (red lines); (3) the model linear in $\log _{10} k_{\mathrm{M}}$ plus the linear bsplines (knots at 4.9 and 7.2) for $\log _{10} K_{\mathrm{OW}}$ (green lines); (4) the model linear in $\log _{10} k_{\mathrm{M}}$ plus the linear b-splines (knots at 4.9 and 7.2) for $\log _{10} K_{\mathrm{OW}}$ for marine and freshwater food webs (dark blue lines); and (5) the model linear in $\log _{10} k_{\mathrm{M}}$ plus the linear b-splines (knots at 4.9 and 7.2) for $\log _{10} K_{\mathrm{OW}}$ for marine temperate whole, marine temperate cold, marine arctic whole, and freshwater temperate cold food webs (cyan lines). There is substantially more support for model (5) with both predictors for the four groups of food webs compared to the other four models based on the large $\triangle \mathrm{AIC}$ at all quantiles. (B) Differences in $R^{1}$ coefficients of determination for the same five models. A greater proportion of variation in $\log _{10}$ TMF was explained by model (5) with both predictors for the four food web groups, and more variation explained at lower compared to higher quantiles.
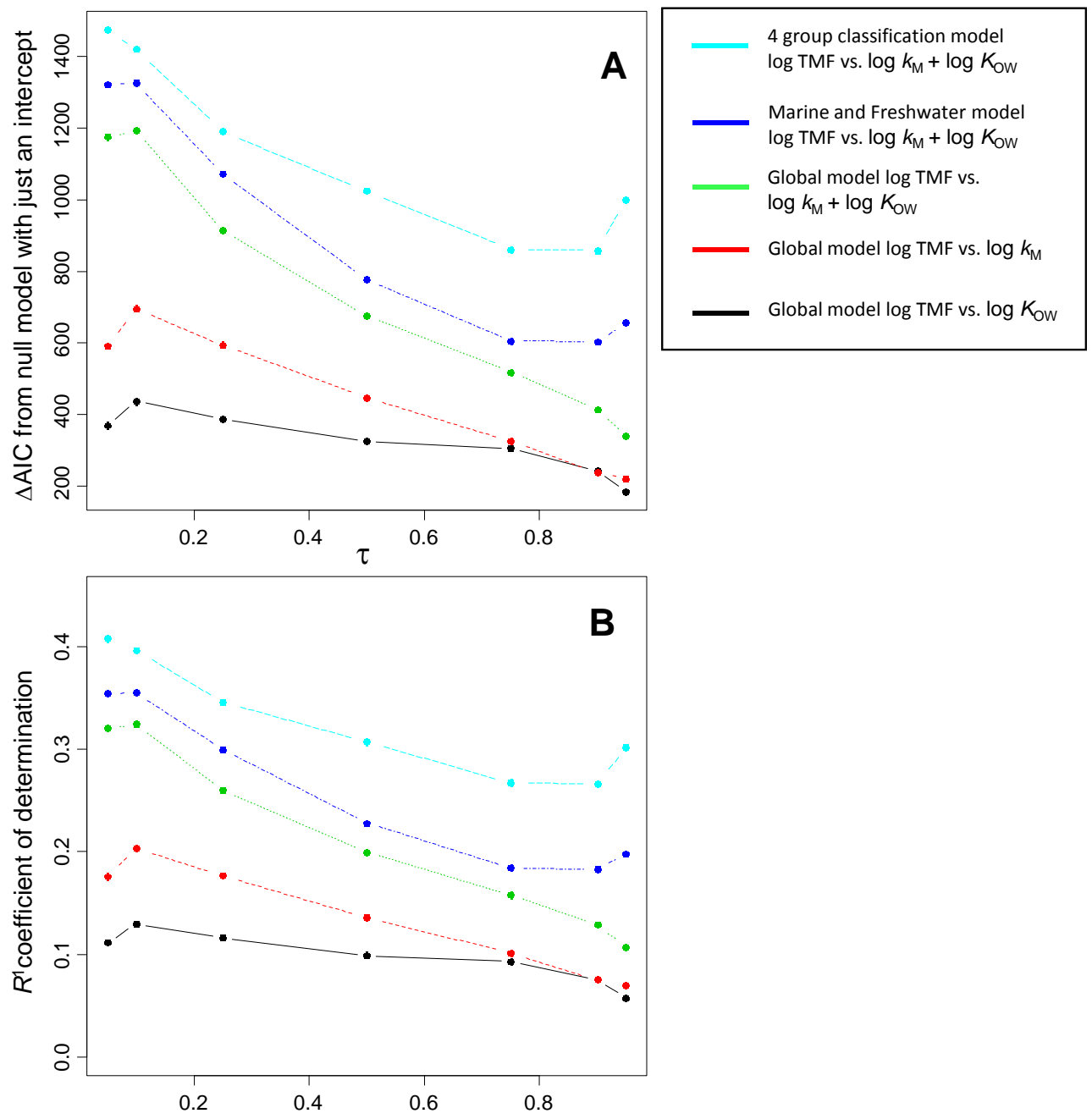
Figure S6. The distribution and density of studies included in this meta-analysis $(n=69)$. Black latitudinal lines show the delineations for tropical $\left(0-30^{\circ}\right)$, temperate $\left(30-60^{\circ}\right)$, and Arctic (60$90^{\circ}$ ) regions. The map was created in R (84) using the "ggplot2" and "maps" packages. Map coordinates for study sites were extracted directly from papers or were determined using Google Maps.

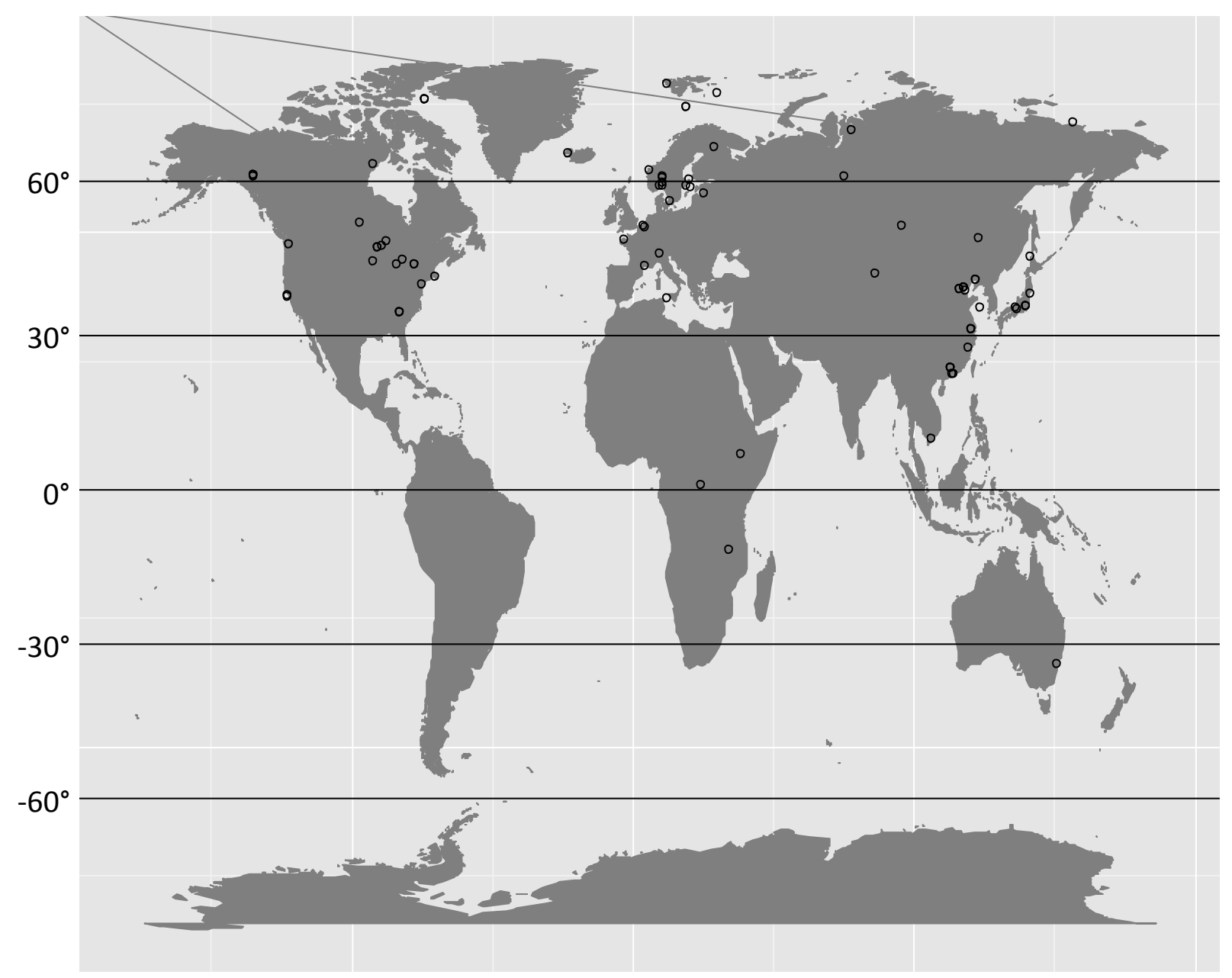


Figure S7. Box and whisker plots of TMF values by compound class. Boxes represent interquartile range, solid line is the median, whiskers indicate the $10^{\text {th }}$ and 90th percentiles, and open circles are outliers. Numbers inside boxes indicate sample size. Red vertical line shows TMF = 1; values > 1 indicates biomagnification. Compound classes are from Newman 2009 (85).

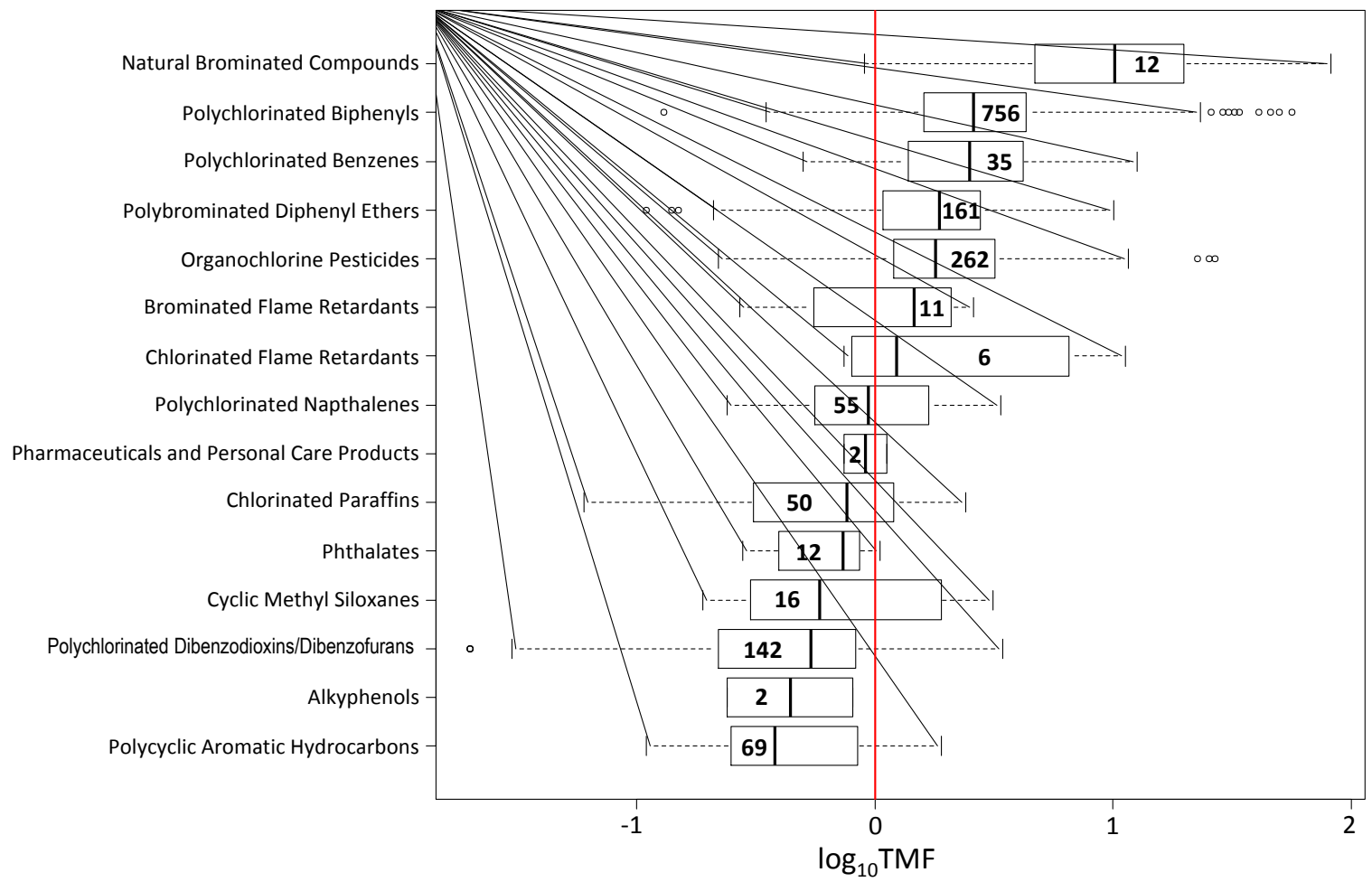


Figure S8. Estimated quantiles $(0.95=$ upper red, $0.50=$ black, $0.05=$ lower red lines $)$ of $\log _{10}$ trophic magnification factor (TMF) as a linear function of $k_{\mathrm{M}}$ (left panels); a linear b-spline function of $\log _{10} K_{O W}$ with knots at $\log _{10} K_{O W}=4.9$ and 7.2 (center panel); and for linear b-spline function of $\log _{10} \mathrm{~K}_{\mathrm{OW}}$ (same knots) plus linear $k_{\mathrm{M}}$ (right panel) for various combinations of ecosystem type, food web category, and climates. Black horizontal lines in $k_{\mathrm{M}}$ and $K_{O W}$ plots show TMF $=1$; values $>1$ indicate biomagnification. For TMF models combining $k_{\mathrm{M}}$ and $K_{O W}$ (right panel), the colored 3-D surface shows the median $(\tau=0.50)$ TMF response, and the gray 3 -D grids are the 0.95 and 0.05 quantiles. Light green, yellow, orange, and red shading indicate TMF > 1 . 
Marine $(n=753)$
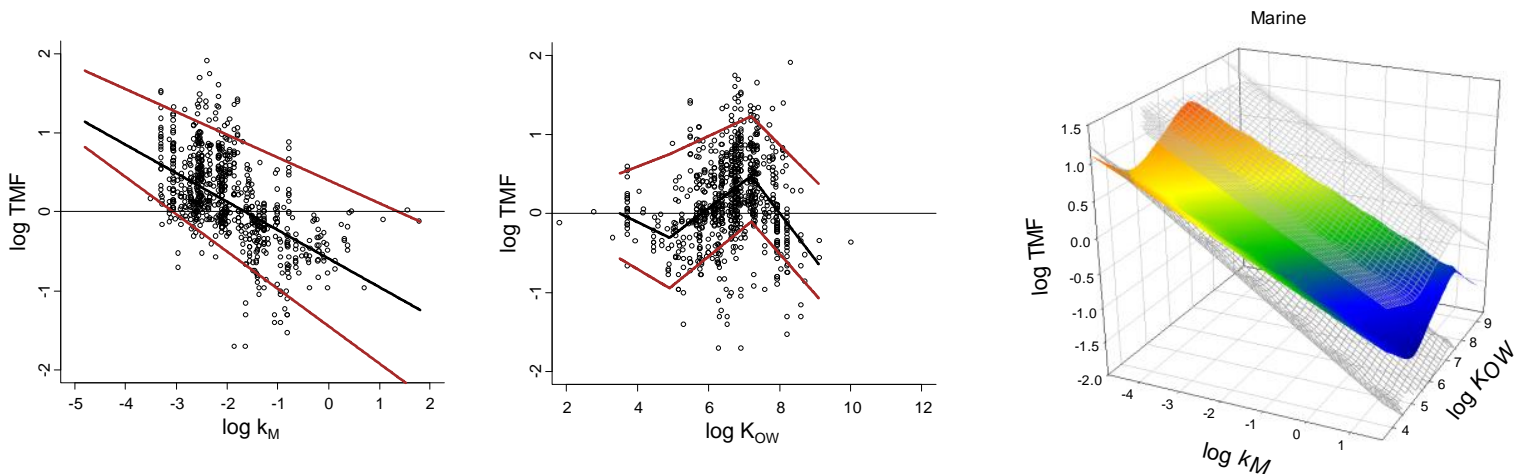

Freshwater $(n=780)$
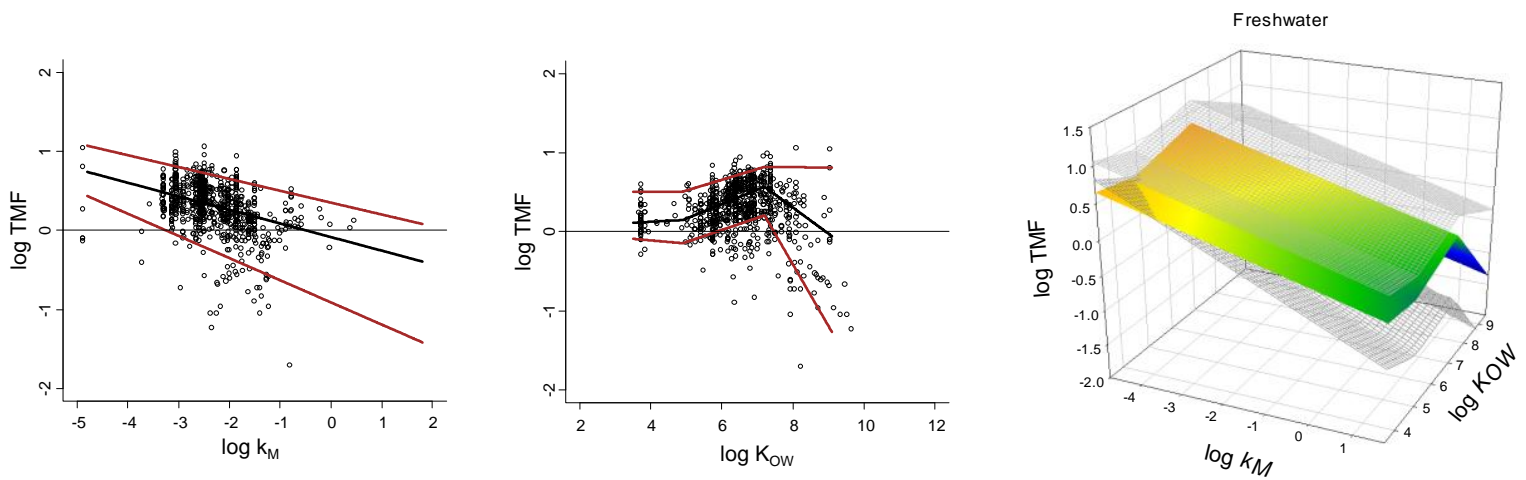

Marine Arctic Whole $(n=132)$
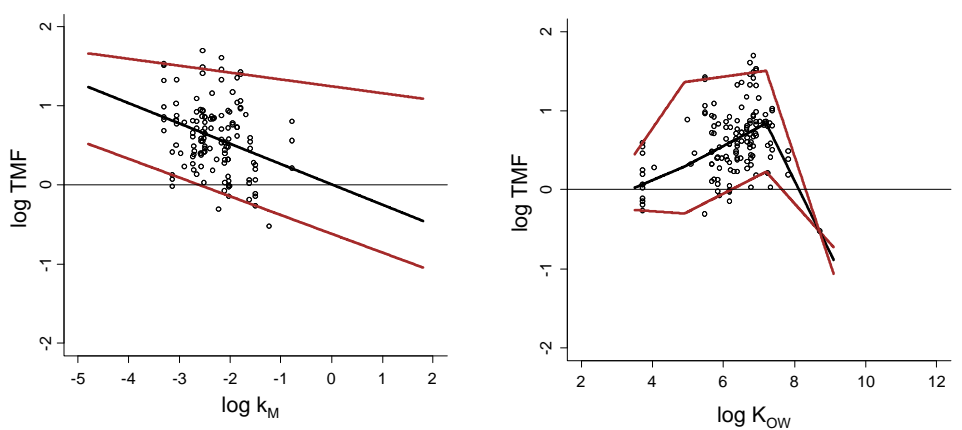

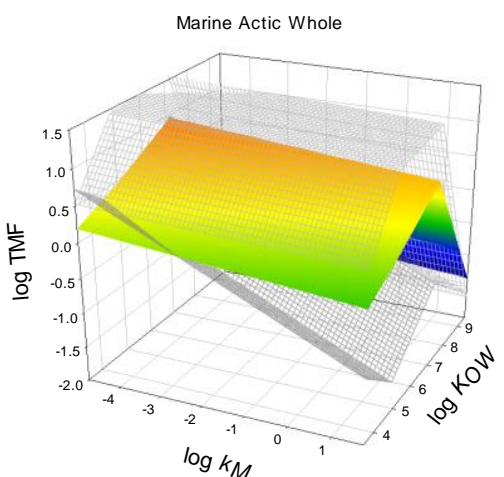

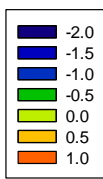


Figure S8 (cont)

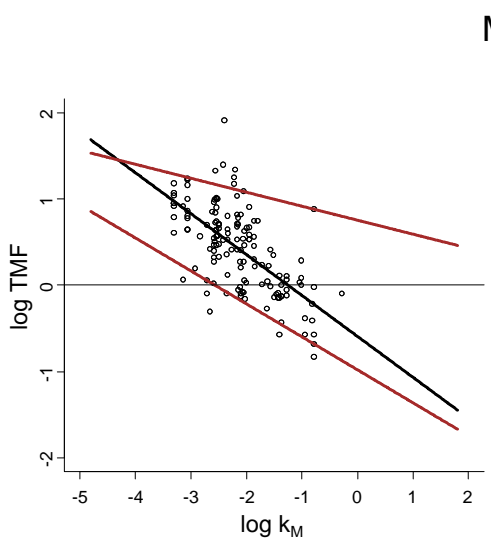

Marine Temperate Whole $(n=144)$
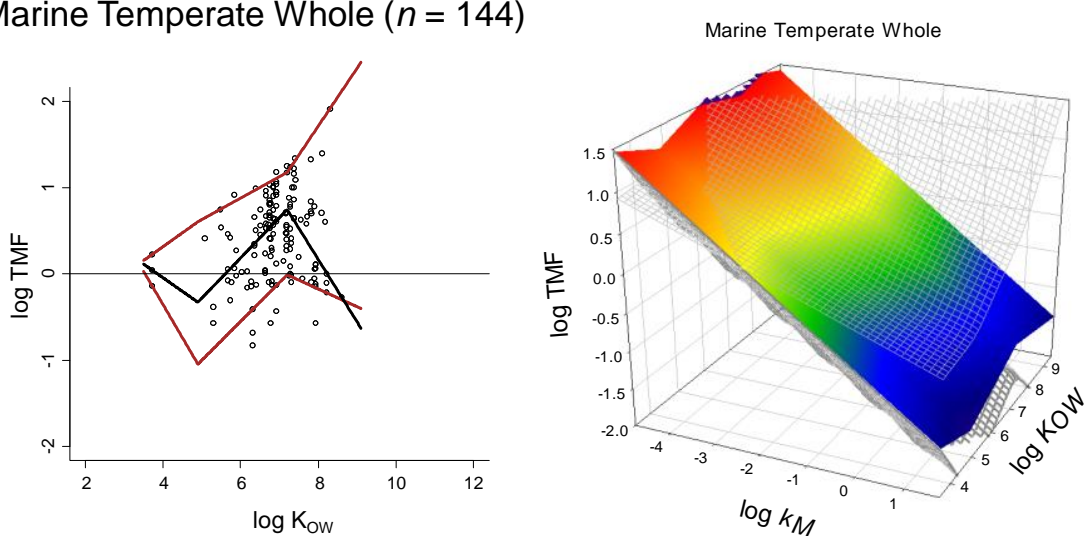

Marine Temperate Cold $(n=454)$
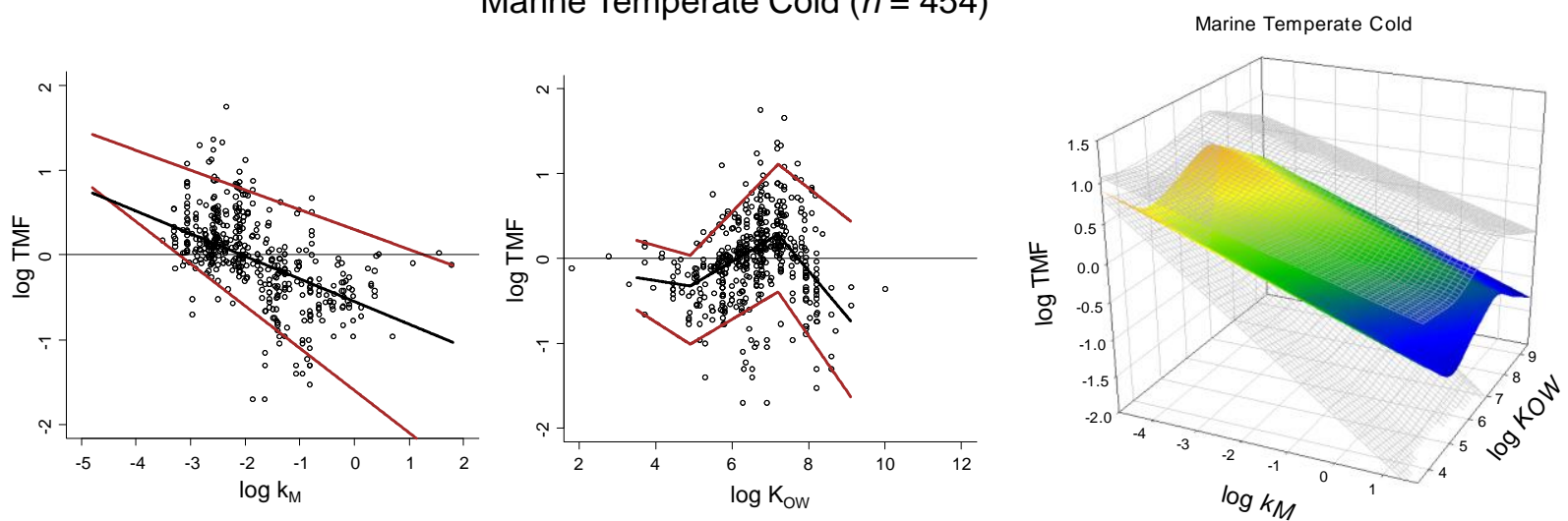

Freshwater Temperate Cold $(n=718)$
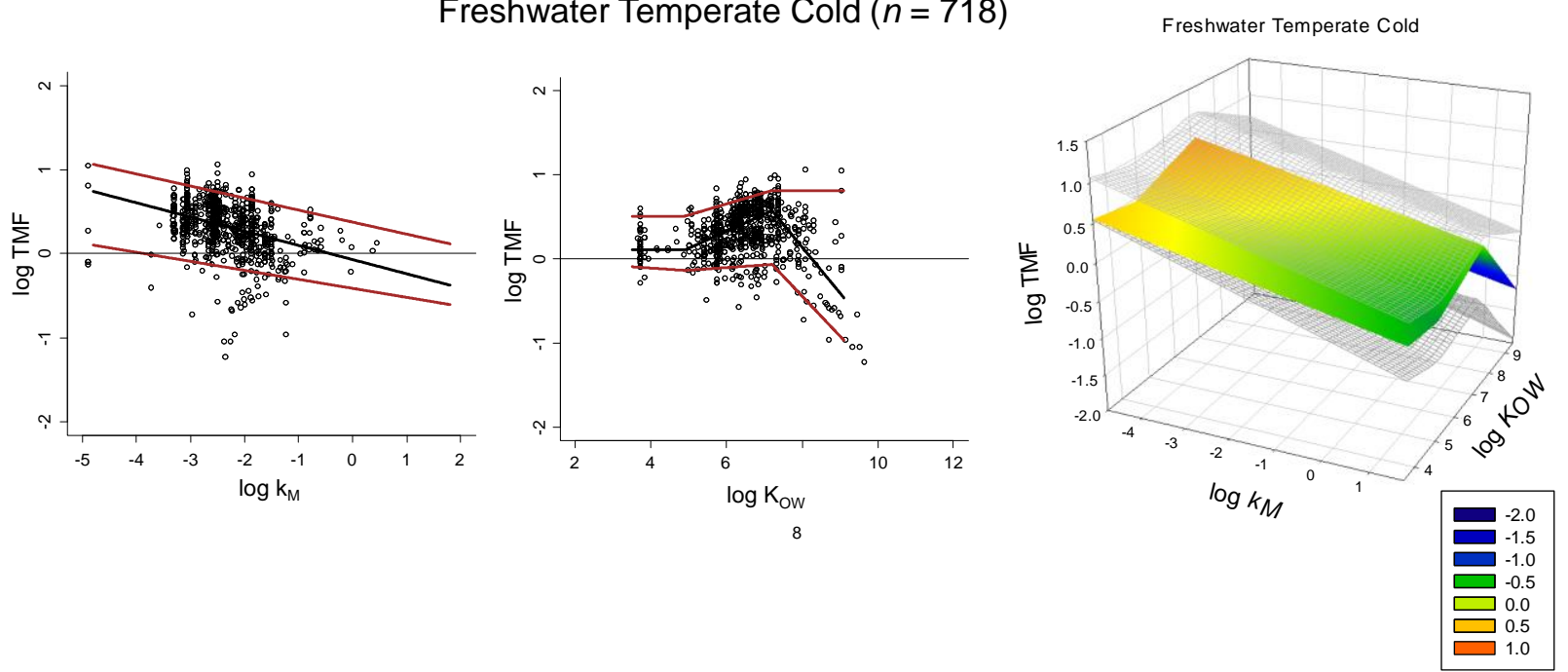
Figure S9. Log TMF vs. latitude for food webs containing only ectotherms (“cold", solid circles) and ectotherms plus endotherms ("whole", open circles) for five compounds: DDE (A), PCB-118 (B), PCB-138 (C), PCB-153 (D), PCB-180 (E). ANCOVA outputs are provided with interactions when significant. When interactions were not significant, the interaction term was removed and the model re-analysed.

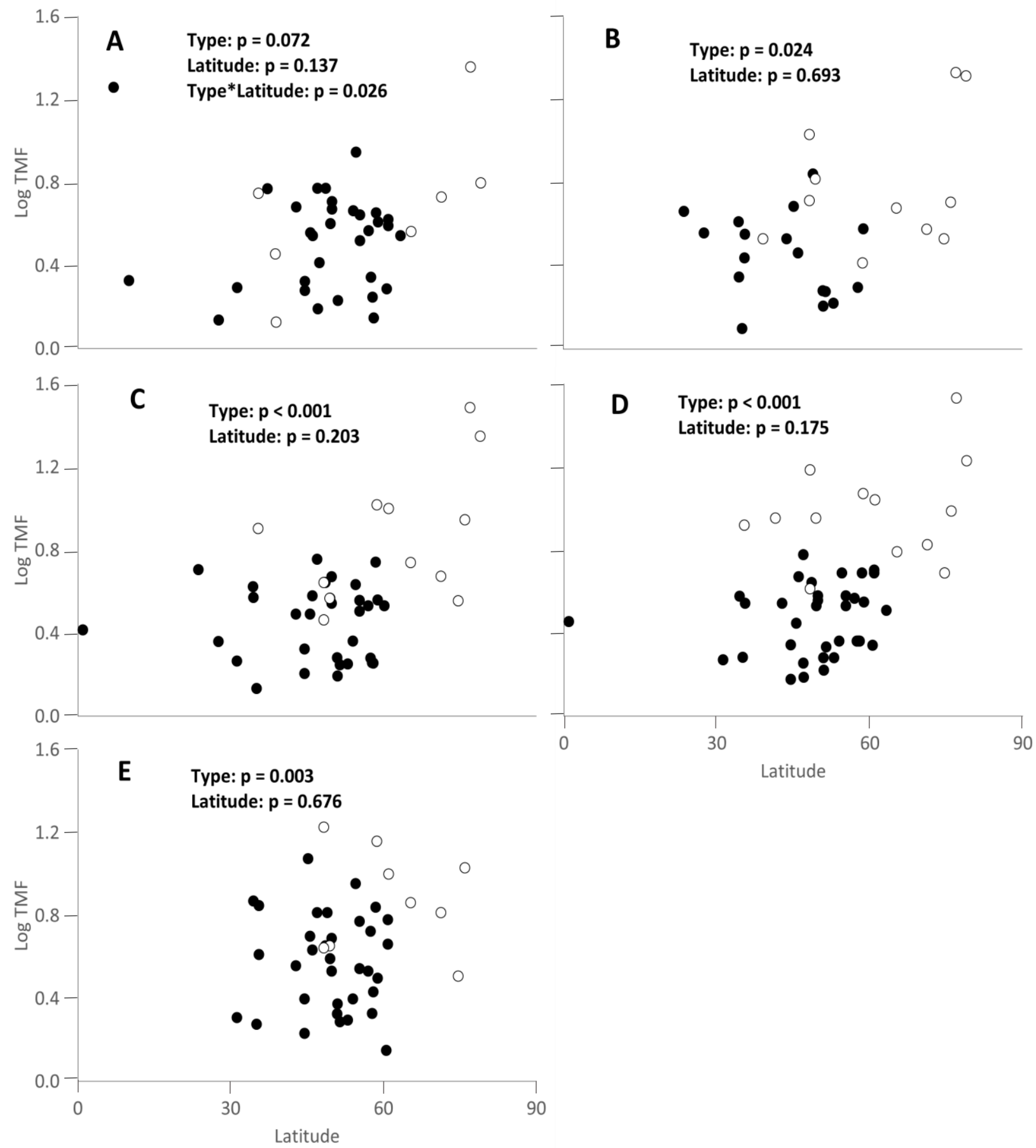


Figure S10. TMFs for chemicals in food webs containing only ectotherms ("cold") and ectotherms plus endotherms ("whole"). A. Mean TMFs for chemicals with at least 5 observations in both food web types. Text labels identify individual chemicals in the plot. B. Mean TMF for all chemicals with at least 1 observation labeled by category: BFR = brominated flame retardents; $\mathrm{OP}=$ organchlorine pesticides; $\mathrm{PB}=$ polychlorinated benzenes; $\mathrm{PBDE}=$ polybrominated diphenyl ethers; $\mathrm{PBDF}=$ polychlorinated benzodioxins and benzofurans; $\mathrm{PCB}=$ polychlorinated biphenyls. Dotted line represents the 1:1 relationship.

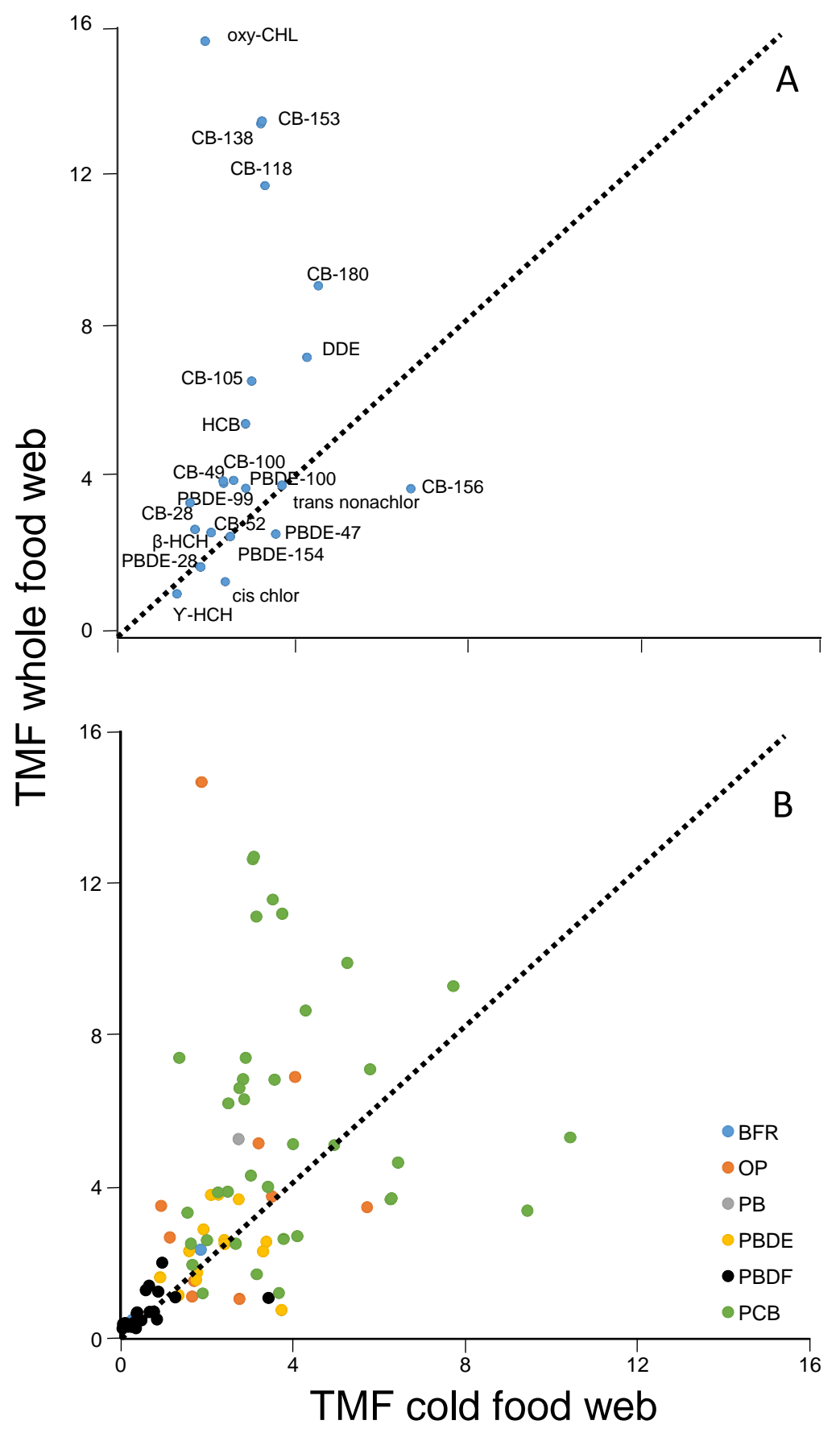




\section{Literature Cited}

(1) Lavoie, R. A.; Jardine, T. D.; Chumchal, M. M.; Kidd, K. A.; Campbell, L. M. Biomagnification of mercury in aquatic food webs: a worldwide meta-analysis. Environ. Sci. Technol. 2013, 47, 13385-13394.

(2) Borga, K.; Kidd, K. A.; Muir, D. C. G.; Berglund, O.; Conder, J. M.; Gobas, F. A. P. C.; Kucklick, J. R.; Malm, O.; Powell, D. E. Trophic magnification factors: considerations of ecology, ecosystems, and study design. Integr. Environ. Assess. Manage. 2012, 8, 64-84. Jardine, T. D.; Kidd, K. A.; Fisk, A. T. Applications, considerations, and sources of uncertainty when using stable isotope analysis in ecotoxicology. Environ. Sci. Technol. 2006, 40, 7501-7511.

(4) Fisk, A. T.; Hobson, K. A.; Norstrom, R. J. Influence of chemical and biological factors on trophic transfer of persistent organic pollutants in the Northwater Polynya marine food web. Environ. Sci. Technol. 2001, 35, 732-738.

(5) Hebert, C. E.; Keenleyside, K. A. To normalize or not to normalize? Fat is the question. Environ. Toxicol. Chem. 1995, 14, 801-807.

(6) Burkhard, L. P.; Borga, K.; Powell, D. E.; Leonards, P.; Muir, D. C. G.; Parkerton, T. F.; Woodburn, K. B. Improving the quality and scientific understanding of Trophic Magnification Factors (TMFs). Environ. Sci. Technol. 2013, 47, 1186-1187.

(7) Conder, J. M.; Gobas, F. A. P. C.; Borga, K.; Muir, D. C. G.; Powell, D. E. Use of trophic magnification factors and related measures to characterize bioaccumulation potential of chemicals. Integr. Environ. Assess. Manage. 2012, 8, 85-97.

(8) Houde, M.; Bujas, T. A. D.; Small, J.; Wells, R. S.; Fair, P. A.; Bossart, G. D.; Solomon, K. R.; Muir, D. C. G. Biomagnification of perfluoroalkyl compounds in the bottlenose dolphin (Tursiops truncatus) food web. Environ. Sci. Technol. 2006, 40, 4138-4144.

(9) Hawker, D. W.; Connell, D. W. Octanol-water partition coefficients of polychlorinated biphenyl congeners. Environ. Sci. Technol. 1988, 22, 382-387.

(10) Zhou, W.; Zhai, Z. C.; Wang, Z. Y.; Wang, L. S. Estimation of n-octanol/water partition coefficients (K-ow) of all PCB congeners by density functional theory. Journal of Molecular Structure-Theochem 2005, 755, 137-145.

(11) Kelly, B. C.; Ikonomou, M. G.; Blair, J. D.; Gobas, F. A. P. C. Hydroxylated and methoxylated polybrominated diphenyl ethers in a Canadian Arctic marine food web. Environ. Sci. Technol. 2008, 42, 7069-7077.

(12) Fisk, A. T.; Rosenberg, B.; Cymbalisty, C. D.; Stern, G. A.; Muir, D. C. G. Octanol/water partition coefficients of toxaphene congeners determined by the "slow-stirring" method. Chemosphere 1999, 39, 2549-2562.

(13) Arnot, J. A.; Mackay, D.; Parkerton, T. F.; Bonnell, M. A database of fish biotransformation rates for organic chemicals. Environ. Toxicol. Chem. 2008, 27, 22632270.

(14) Houde, M., et al. Influence of lake characteristics on the biomagnification of persistent organic pollutants in lake trout food webs. Environ. Toxicol. Chem. 2008, 27, 2169-2178.

(15) Hallanger, I. G.; Warner, N. A.; Ruus, A.; Evenset, A.; Christensen, G. N.; Herzke, D.; Gabrielsen, G. W.; Borga, K. Seasonality in contaminant accumulation in Arctic marine pelagic food webs using trophic magnification factor as a measure of bioaccumulation. Environ. Toxicol. Chem. 2011, 30, 1026-1035. 
(16) Hu, J.; Jin, F.; Wan, Y.; Yang, M.; An, L.; An, W.; Tao, S. Trophodynamic behavior of 4-nonylphenol and nonylphenol polyethoxylate in a marine aquatic food web from Bohai Bay, North China: Comparison to DDTs. Environ. Sci. Technol. 2005, 39, 4801-4807.

(17) Takeuchi, I.; Miyoshi, N.; Mizukawa, K.; Takada, H.; Ikemoto, T.; Omori, K.; Tsuchiya, $\mathrm{K}$. Biomagnification profiles of polycyclic aromatic hydrocarbons, alkylphenols and polychlorinated biphenyls in Tokyo Bay elucidated by $\delta 13 \mathrm{C}$ and $\delta 15 \mathrm{~N}$ isotope ratios as guides to trophic web structure. Mar. Pollut. Bull. 2009, 58, 663-671.

(18) Brisebois, A. R. Relationship between the Bioconcentration Factor (BCF), the Bioaccumulation Factor (BAF), and the Trophic Magnification Factor (TMF). Simon Fraser University, 2013.

(19) Haukas, M.; Hylland, K.; Nygard, T.; Berge, J. A.; Mariussen, E. Diastereomer-specific bioaccumulation of hexabromocyclododecane (HBCD) in a coastal food web, Western Norway. Sci. Total Environ. 2010, 408, 5910-5916.

(20) Tomy, G. T.; Pleskach, K.; Oswald, T.; Halldorson, T.; Helm, P. A.; Macinnis, G.; Marvin, C. H. Enantioselective bioaccumulation of hexabromocyclododecane and congener- specific accumulation of brominated diphenyl ethers in an eastern Canadian Arctic marine food web. Environ. Sci. Technol. 2008, 42, 3634-3639.

(21) Wu, J.; Guan, Y.; Zhang, Y.; Luo, X.; Zhi, H.; Chen, S.; Mai, B. Trophodynamics of hexabromocyclodecanes and several other non-PBDE brominated flame retardants in a freshwater food web. Environ. Sci. Technol. 2010, 44, 5490-5495.

(22) Tomy, G. T.; Pleskach, K.; Ismail, N.; Whittle, D. M.; Helm, P. A.; Sverko, E.; Zaruk, D.; Marvin, C. H. Isomers of dechlorane plus in Lake Winnipeg and Lake Ontario food webs. Environ. Sci. Technol. 2007, 41, 2249-2254.

(23) Zhang, Y.; Luo, X.; Wu, J.; Liu, J.; Wang, J.; Chen, S.; Mai, B. Contaminant pattern and bioaccumulation of legacy and emerging organhalogen pollutants in the aquatic biota from an e-waste recycling region in South China. Environ. Toxicol. Chem. 2010, 29, 852859.

(24) Wu, J.; Zhang, Y.; Luo, X.; Wang, J.; Chen, S.; Guan, Y.; Mai, B. Isomer-specific bioaccumulation and trophic transfer of dechlorane plus in the freshwater food web from a highly contaminated site, South China. Environ. Sci. Technol. 2010, 44, 606-611.

(25) Houde, M.; Muir, D. C. G.; Tomy, G. T.; Whittle, D. M.; Teixeira, C.; Moore, S. Bioaccumulation and trophic magnification of short- and medium-chain chlorinated paraffins in food webs from Lake Ontario and Lake Michigan. Environ. Sci. Technol. 2008, 42, 3893-3899.

(26) Borga, K.; Fjeld, E.; Kierkegaard, A.; McLachlan, M. S. Consistency in trophic magnification factors of cyclic methyl siloxanes in pelagic freshwater food webs leading to Brown Trout. Environ. Sci. Technol. 2013, 47, 14394-14402.

(27) Powell, D. E. Bioaccumulation and trophic transfer of cyclic volatile methylsiloxane (cVMS) materials in the aquatic marine food webs of the inner and outer Oslofjord, Norway; Centre Europeen des Silicones (CES): Auburn, MI, 2010.

(28) Powell, D. E.; Woodburn, K. B. Trophic dilution of cyclic volatile methylsilozane (cVMS) materials in a temperate freshwater lake; Centre Europeen des Silocones (CES): Auburn, Michigan, 2009.

(29) Borga, K.; Fjeld, E.; Kierkegaard, A.; McLachlan, M. S. Food web accumulation of cyclic siloxanes in Lake Mjosa, Norway. Environ. Sci. Technol. 2012, 46, 6347-6354. 
(30) Losada, S.; Roach, A.; Roosens, L.; Santos, F. J.; Galceran, M. T.; Vetter, W.; Neels, H.; Covaci, A. Biomagnification of anthropogenic and naturally-produced organobrominated compounds in a marine food web from Sydney Harbour, Australia. Environ. Internat. 2009, 35, 1142-1149.

(31) Pangallo, K. C.; Reddy, C. M. Marine natural products, the halogenated 1'-methyl-1,2'bipyrroles, biomagnify in a northwestern Atlantic food web. Environ. Sci. Technol. 2010, 44, 5741-5747.

(32) Tittlemier, S. A.; Fisk, A. T.; Hobson, K. A.; Norstrom, R. J. Examination of the bioaccumulation of halogenated dimethyl bipyrroles in an Arctic marine food web using stable nitrogen isotope analysis. Environ. Pollut. 2002, 116, 85-93.

(33) Wang, D.-Q.; Yu, Y.-X.; Zhang, X.-Y.; Zhang, S.-H.; Pang, Y.-P.; Zhang, X.-L.; Yu, Z.Q.; Wu, M.-H.; Fu, J.-M. Polycyclic aromatic hydrocarbons and organochlorine pesticides in fish from Taihu Lake: Their levels, sources, and biomagnification. Ecotoxicol. Environ. Saf. 2012, 82, 63-70.

(34) Muir, D. C. G.; Whittle, D. M.; De Vault, D. S.; Bronte, C. R.; Karlsson, H.; Backus, S.; Teixeira, C. Bioaccumulation of toxaphene congeners in the Lake Superior food web. $J$. Great Lakes Res. 2004, 30, 316-340.

(35) Nfon, E.; Cousins, I. T.; Broman, D. Biomagnification of organic pollutants in benthic and pelagic marine food chains from the Baltic Sea. Sci. Total Environ. 2008, 397, 190204.

(36) Hoekstra, P. F.; O'Hara, T. M.; Fisk, A. T.; Borga, K.; Solomon, K. R.; Muir, D. C. G. Trophic transfer of persistent organochlorine contaminants (OCs) within an Arctic marine food web from the southern Beaufort-Chukchi Seas. Environ. Pollut. 2003, 124, 509-522. Hop, H.; Borga, K.; Gabrielsen, G. W.; Kleivane, 1.; Skaare, J. U. Food web magnification of persistent organic pollutants in poikilotherms and homeotherms from the Barents Sea. Environ. Sci. Technol. 2002, 36, 2589-2597.

(38) Ikemoto, T.; Tu, N. P. C.; Watanabe, M. X.; Okuda, N.; Omori, K.; Tanabe, S.; Tuyen, B. C.; Takeuchi, I. Analysis of biomagnification of persistent organic pollutants in the aquatic food web of the Mekong Delta, South Vietnam using stable carbon and nitrogen isotopes. Chemosphere 2008, 72, 104-114.

(39) Kucklick, J. R.; Baker, J. E. Organochlorines in Lake Superior's food web. Environ. Sci. Technol. 1998, 32, 1192-1198.

(40) Skarphedinsdottir, H.; Gunnarsson, K.; Gudmundsson, G. A.; Nfon, E. Bioaccumulation and biomagnification of organochlorines in a marine food web at a pristine site in Iceland. Arch. Environ. Contam. Toxicol. 2010, 58, 800-809.

(41) Hu, G.; Dai, J.; Mai, B.; Luo, X.; Cao, H.; Wang, J.; Li, F.; Xu, M. Concentrations and accumulation features of organochlorine pesticides in the Baiyangdian lake freshwater food web of North China. Arch. Environ. Contam. Toxicol. 2010, 58, 700-710.

(42) Verhaert, V.; Covaci, A.; Bouillon, S.; Abrantes, K.; Musibono, D.; Bervoets, L.; Veryheyen, E.; Blust, R. Baseline levels and trophic transfer of persistent organic pollutants in sediments and biota from the Congo River Basin (DR Congo). Environ. Internat. 2013, 59, 290-302.

(43) Villa, S.; Bizzotto, E. C.; Vighi, M. Persistent organic pollutant in a fish community of a sub-alpine lake. Environ. Pollut. 2011, 159, 932-939.

(44) Hoekstra, P. F.; O'Hara, T. M.; Fisk, A. T.; Borga, K.; Solomon, K. R.; Muir, D. C. G. Trophic transfer of persistent orgranochlorine contaminants (OCs) within an Arctic 
marine food web from the southern Beaufort-Chukchi Seas. Environ. Pollut. 2003, 124, 509-522.

(45) Moisey, J.; Fisk, A. T.; Hobson, K. A.; Norstrom, R. J. Hexachlorocyclohexane (HCH) isomers and chiral signatures of $\alpha-\mathrm{HCH}$ in the Arctic marine food web of the Northwater Polynya. Environ. Sci. Technol. 2001, 35, 1920-1927.

(46) Olsson, A.; Valters, K.; Burreau, S. Concentrations of organochlorine substances in relation to fish size and trophic position: A study on perch (Perca fluviatilis L.). Environ. Sci. Technol. 2000, 34, 4878-4886.

(47) Van Ael, E.; Covaci, A.; Das, K.; Lepoint, G.; Blust, R.; Bervoets, L. Factors influencing the bioaccumulation of persistent organic pollutants in food webs of the Scheldt Estuary. Environ. Sci. Technol. 2013, 47, 11221-11231.

(48) Byun, G.-H.; Moon, H.-B.; Choi, J.-H.; Hwang, J.; Kang, C.-K. Biomagnification of persistent chlorinated and brominated contaminants in food web components of the Yellow Sea. Mar. Pollut. Bull. 2013, 73, 210-219.

(49) Corsolini, S.; Sara, G.; Borghesi, N.; Focardi, S. HCB, p, p'-DDE and PCB ontogenetic transfer and magnification in Bluefin Tuna (Thunnus thynnus) from the Mediterranean Sea. Environ. Sci. Technol. 2007, 41, 4227-4233.

(50) Kidd, K. A.; Hesslein, R. H.; Ross, B. J.; Koczanski, K.; G.R., S.; Muir, D. C. G. Bioaccumulation of organochlorines through a remote freshwater food web in the Canadian Arctic. Environ. Pollut. 1998, 102, 91-103.

(51) Kidd, K. A.; Schindler, D. W.; Hesslein, R. H.; Muir, D. C. G. Effects of trophic position and lipid on organochlorine concentrations in fishes from subarctic lakes in Yukon Territory. Can. J. Fish. Aquat. Sci. 1998, 55, 869-881.

(52) Zhang, X.; Xu, Q.; Man, S.; Zeng, X.; Yu, Y.; Pang, Y.; Sheng, G.; Fu, J. Tissue concentrations, bioaccumulation, and biomagnification of synthetic musks in freshwater fish from Taihu Lake, China. Environmental Science and Pollution Research 2013, 20, 311-322.

(53) Mackintosh, C. E.; Maldonado, J.; Hongwu, J.; Hoover, N.; Chong, A.; Ikonomou, M. G.; Gobas, F. A. P. C. Distribution of phthalate esters in a marine aquatic food web: comparison to polychlorinated biphenyls. Environ. Sci. Technol. 2004, 38, 2011-2020.

(54) Zhang, K.; Wan, Y.; Jones, P. D.; Wiseman, S.; Giesy, J. P.; Hu, J. Occurrences and fates of hydroxylated polybrominated diphenyl ethers in marine sediments in relation to trophodynamics. Environ. Sci. Technol. 2012, 46, 2148-2155.

(55) Mizukawa, K.; Takada, H.; Takeuchi, I.; Ikemoto, T.; Omori, K.; Tsuchiya, K. Bioconcentration and biomagnification of polybrominated diphenyl ethers (PBDEs) through lower-trophic-level coastal marine food web. Mar. Pollut. Bull. 2009, 58, 1217 1224.

(56) Mizukawa, K.; Yamada, T.; Matsuo, H.; Takeuchi, I.; Tsuchiya, K.; Takada, H. Biomagnification and debromination of polybrominated diphenyl ethers in a coastal ecosystem in Tokyo Bay. Sci. Total Environ. 2013, 449, 401-409.

(57) Yu, Y.-X.; Zhang, S.-H.; Huang, N.-B.; Li, J.-L.; Pang, Y.-P.; Zhang, X.-Y.; Yu, Z.-Q.; $\mathrm{Xu}, \mathrm{Z} . \mathrm{-G}$. Polybrominated diphenyl ethers and polychlorinated biphenyls in freshwater fish from Taihu Lake, China: Their levels and the factors that influence biomagnification. Environ. Toxicol. Chem. 2012, 31, 542-549.

(58) Hu, G.-c.; Dai, J.-y.; Xu, Z.-c.; Luo, X.-j.; Cao, H.; Wang, J.-s.; Mai, B.-X.; Xu, M.-q. Bioaccumulation behavior of polybrominated diphenyl ethers (PBDEs) in the freshwater 
food chain of Baiyangdian Lake, North China. Environmental International 2010, 36, 309-315.

(59) Wan, Y.; Hu, J.; Zhang, K.; An, L. Trophodynamics of polybrominated diphenyl ethers in the marine food web of Bohai Bay, North China. Environ. Sci. Technol. 2008, 42, 1078-1083.

(60) Wu, J.; Luo, X.; Zhang, Y.; Yu, M.; Chen, S.; Mai, B.; Yang, Z. Biomagnification of polybrominated diphenyl ethers (PBDEs) and polychlorinated biphenyls in a highly contaminated freshwater food web from South China. Environ. Pollut. 2009, 157, 904909.

(61) Yu, M.; Luo, X.; Wu, J.; Chen, S.; Mai, B. Bioaccumulation and trophic transfer of polybrominated diphenyl ethers (PBDEs) in biota from the Pearl River Estuary, South China. Environmental International 2009, 35, 1090-1095.

(62) Zhang, K.; Wan, Y.; An, L.; Hu, J. Trophodynamics of polybrominated diphenyl ethers and methoxylated polybrominated diphenyl ethers in a marine food web. Environ. Toxicol. Chem. 2010, 29, 2792-2799.

(63) Burreau, S.; Zebuhr, Y.; Broman, D.; Ishaq, R. Biomagnification of polychlorinated biphenyls (PCBs) and polybrominated diphenyl ethers (PBDEs) studied in pike (Esox lucius), perch (Perca fluviatilis) and roach (Rutilus rutilus) from the Baltic Sea. Chemosphere 2004, 55, 1043-1052.

(64) Kuo, Y.-M.; Sepulveda, M. S.; Hua, I.; Ochoa-Acuna, H. G.; Sutton, T. M. Bioaccumulation and biomagnification of polybrominated diphenyl ethers in a food web of Lake Michigan. Ecotoxicology 2010, 19, 623-634.

(65) Jarman, W. M.; Hobson, K. A.; Sydeman, W. J.; Bacon, C. E.; Maclaren, E. B. Influence of trophic position and feeding location on contaminant levels in the Gulf of the Farallones food web revealed by stable isotope analysis. Environ. Sci. Technol. 1996, 30, 654-660.

(66) Takahashi, S.; Oshihoi, T.; Ramu, K.; Isobe, T.; Ohmori, K.; Kubodera, T.; Tanabe, S. Organohalogen compounds in deep-sea fishes from the western North Pacific, offTohoku, Japan: Contamination status and bioaccumulation profiles. Mar. Pollut. Bull. 2010, 60, 187-196.

(67) Broman, D.; Naf, C.; Rolff, C.; Zebuhr, Y.; Fry, B.; John, H. Using ratios of stable nitrogen isotopes to estimate bioaccumulation and flux of polychlorinated dibenzo-pdioxins (PCDDs) and dibenzofurans (PCDFs) in two food chains from the northern baltic. Environ. Toxicol. Chem. 1992, 11, 331-345.

(68) Jarman, W. M.; Sydeman, W. J.; Hobson, K. A.; Bergqvist, P.-A. Relationship of polychlorinated dibenzo-p-dioxin and polychlorinated dibenzofuran levels to stablenitrogen isotope abundance in marine birds and mammals in coastal California. Environ. Toxicol. Chem. 1997, 16, 1010-1013.

(69) Kang, Y.-S.; Jin, J.; Yamamuro, M.; Masunaga, S.; Nakanishi, J. Dynamics of PCDDs/DFs and coplanar-PCBs in aquatic food chains from Lake Shinji and Tokyo Bay.

(70) Naito, W.; Jin, J.; Kang, Y.-S.; Yamamuro, M.; Masunaga, S.; Nakanishi, J. Dynamics of PCDDs / DFs and coplanar-PCBs in an aquatic food chain of Tokyo Bay. Chemosphere 2003, 53, 347-362.

(71) Wan, Y.; Hu, J.; Yang, M.; An, L.; An, W.; Jin, X.; Hattori, T.; Itoh, M. Characterization of trophic transfer for polychlorinated dibenzo-p-dioxins, dibenzofurans, non- and mono- 
ortho polychlorinated biphenyls in the marine food web of Bohai Bay, North China. Environ. Sci. Technol. 2005, 39, 2417-2425.

(72) Ruus, A.; Berge, J. A.; Bergstad, O. A.; Knutsen, J. A.; Hylland, K. Disposition of polychlorinated dibenzo-p-dioxins (PCDDs) and polychlorinated dibenzofurans (PCDFs) in two Norwegian epibenthic marine food webs. Chemosphere 2006, 62, 1856-1868.

(73) Matsuo, H.; Kawano, M.; Omori, K.; Nakajima, K.; Takeuchi, I. Stable isotope-guided analysis of congener-specific PCB concentrations in a Japanese coastal food web. Mar. Pollut. Bull. 2009, 58, 1615-1623.

(74) Walters, D. M., Twelve-Mile Creek Unpublished Data. In United States Geological Survey.

(75) Walters, D. M.; Mills, M. A.; Cade, B. S.; Burkard, L. P. Trophic magnification of PCBs and its relationship to the octanol-water partition coefficient. Environ. Sci. Technol. 2011, 45, 3917-3924.

(76) Gewurtz, S. B.; Gandhi, N.; Stern, G. A.; Franzin, W. G.; Rosenberg, B.; Diamond, M. L. Dynamics of PCBs in the food web of Lake Winnipeg. J. Great Lakes Res. 1999, 32, 712.

(77) Helm, P. A.; Gewurtz, S. B.; Whittle, D. M.; Marvin, C. H.; Fisk, A. T.; Tomy, G. T. Occurrence and biomagnification of polychlorinated naphthalenes and non- and monoortho PCBs in Lake Ontario sediment and biota. Environ. Sci. Technol. 2008, 42, 10241031.

(78) Wong, C. S.; Mabury, S. A.; Whittle, D. M.; Backus, S. M.; Teixeira, C.; De Vault, D. S.; Bronte, C. R.; Muir, D. C. G. Organochlorine compounds in Lake Superior: chiral polychlorinated biphenyls and biotransformation in the aquatic food web. Environ. Sci. Technol. 2004, 38, 84-92.

(79) Bodin, N.; Le Loc'h, F.; Caisey, X.; Le Guellec, A. M.; Abarnou, A.; Loizeau, V.; Latrouite, D. Congener-specific accumulation and trophic transfer of polychlorinated biphenyls in spider crab food webs revealed by stable isotope analysis. Environ. Pollut. 2008, 151, 252-261.

(80) Sobek, A.; McLachlan, M. S.; Borga, K.; Asplund, L.; Lundstedt-Enkel, K.; Polder, A.; Gustafsson, O. A comparison of PCB bioaccumulation factors between an arctic and a temperate marine food web. Sci. Total Environ. 2010, 408, 2753-2760.

(81) Takeuchi, I.; Miyoshi, N.; Mizukawa, K.; Takada, H.; Ikemoto, T.; Omori, K.; Tsuchiya, $\mathrm{K}$. Biomagnification profiles of polycyclic aromatic hydrocarbons, alkylphenols and polychlorinated biphenyls in Tokyo Bay elucidated by $\delta^{13} \mathrm{C}$ and $\delta^{15} \mathrm{~N}$ isotope ratios as guides to trophic web structure. Mar. Pollut. Bull. 2009, 58, 663-671.

(82) Wan, Y.; Jin, X.; Hu, J.; Jin, F. Trophic dilution of polycyclic aromatic hydrocarbons (PAHs) in a marine food web from Bohai Bay, north China. Environ. Sci. Technol. 2007, 41, 3109-3114.

(83) Koenker, R. Additive models for quantile regression: model selection and confidence bandaids. Brazilian Journal of Probability and Statistics 2011, 25, 269-262.

(84) R Development Core Team R: A Language and Environment for Statistical Computing; R Foundation for Statistical Computing, Vienna, Austria. Available at http://www.Rproject.org; 2008.

(85) Newman, M. C. Fundamentals of Ecotoxicology. 3rd ed.; CRC Press: Boca Raton, FL, 2009. 